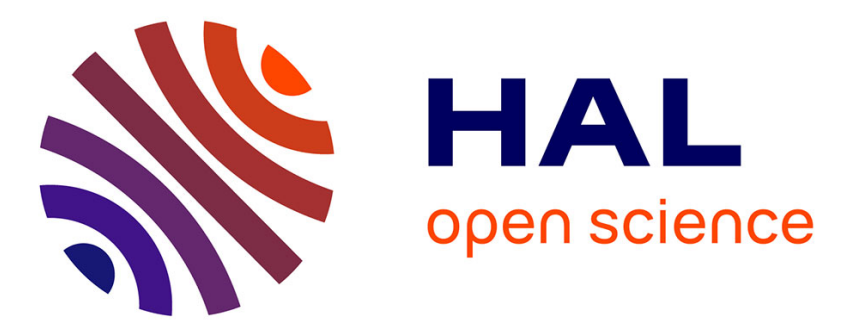

\title{
Divergence-free Wavelets and High Order Regularization
}

\author{
Souleymane Kadri Harouna, Pierre Dérian, Patrick Héas, Etienne Mémin
}

\section{To cite this version:}

Souleymane Kadri Harouna, Pierre Dérian, Patrick Héas, Etienne Mémin. Divergence-free Wavelets and High Order Regularization. International Journal of Computer Vision, 2013, 103 (1), pp.80-99. 10.1007/s11263-012-0595-7 . hal-00646104v2

\section{HAL Id: hal-00646104 \\ https://hal.science/hal-00646104v2}

Submitted on 25 Jan 2012

HAL is a multi-disciplinary open access archive for the deposit and dissemination of scientific research documents, whether they are published or not. The documents may come from teaching and research institutions in France or abroad, or from public or private research centers.
L'archive ouverte pluridisciplinaire HAL, est destinée au dépôt et à la diffusion de documents scientifiques de niveau recherche, publiés ou non, émanant des établissements d'enseignement et de recherche français ou étrangers, des laboratoires publics ou privés. 


\title{
Divergence-free Wavelets and High Order Regularization
}

\author{
S. Kadri-Harouna · P. Dérian · P. Héas · E. Mémin
}

\begin{abstract}
Expanding on a wavelet basis the solution of an inverse problem provides several advantages. First of all, wavelet bases yield a natural and efficient multiresolution analysis which allows defining clear optimization strategies on nested subspaces of the solution space. Besides, the continuous representation of the solution with wavelets enables analytical calculation of regularization integrals over the spatial domain. By choosing differentiable wavelets, accurate high-order derivative regularizers can be efficiently designed via the basis's mass and stiffness matrices. More importantly, differential constraints on vector solutions, such as the divergencefree constraint in physics, can be nicely handled with biorthogonal wavelet bases. This paper illustrates these advantages in the particular case of fluid flow motion estimation. Numerical results on synthetic and real images of incompressible turbulence show that divergencefree wavelets and high-order regularizers are particularly relevant in this context.
\end{abstract}

Keywords Divergence-free wavelets · High order derivatives regularization · optic-flow estimation

\section{Introduction}

Prior models used to solve ill-posed inverse problems such as image restoration, surface reconstruction or optic-flow estimation often involve differential constraints or smoothing term incorporating high-order derivatives [24]. In particular in the context of opticflow estimation, numerous regularization models [10,23]

S. Kadri-Harouna, P. Dérian, P. Héas, E. Mémin

INRIA Rennes-Bretagne Atlantique, Campus universitaire de Beaulieu, 35042 Rennes Cedex, France.

E-mail: $\quad$ Souleymane.Kadri harouna, Pierre.Derian, Patrick.Heas, Etienne.Memin\}@inria.fr

http://www.irisa.fr/fluminance involving penalization of first or second order derivatives of the estimated velocity have been proposed in order to make this estimation problem well-defined, beginning with the original work of Horn and Schunck [10]. However, although estimation of optical flow is an old and well-known problem, it still remains very challenging for deformations metrological issues or for the measurement of fluid flows [9]. Indeed, optical flow standards are in general designed from almost rigid motions consideration and do not include physical considerations on the observed phenomenon occurring eventually at different spatial scales. Several attempts have been carried out in order to introduce some physical constraints, such as incompressibility $[7,26]$ or second order regularization functional preserving blobs of curl and divergence [9].

In addition, inverse problems often involve nonlinear models relating the data term to the unknown. Dealing with non-linearities and the multi-scale structure of motion is particularly challenging for the estimation of deformation fields generated by physical processes. Gaussian multiresolution frameworks [1] or combined integrated/variational formulations [22] have been proposed to circumvent non-linearity and achieve long range displacement estimation from consecutive images. However, the former solutions suffer from a non nested minimization formulation that may impact estimation accuracy, while the latter provide poor results for non-textured images such as images visualizing the transport of a passive scalar. Even worth, as these optic-flow multiresolution schemes work only at very few distinct scales without any explicit connection between scales, estimation of erroneous large scale motions affects severely the whole estimation process. The divergence free constraint ensuing from a volume preserving transformation is in general quite problematic 
to impose as it comes to solve a Poisson equation which is intrinsically a non local problem. Let us note that the combination of local non linear advection diffusion equation with a global divergence free constraint constitutes one of the major difficulties of Navier-Stokes equations numerical simulation. This constraint is imposed through the pressure field which acts as a Lagrangian penalty variable and requires advanced discretization schemes on staggered grid to prevent oscillations [6].

In order to circumvent some of the difficulties associated to traditional optical flow multiresolution approaches, and to enable a relevant multi-scale motion estimation, an optical flow scheme based on the wavelet expansion of the motion field has been introduced by Wu et al. [25]. This technique has however a prohibitive computational cost in $O\left(N^{6}\right)$, where $N$ is the number of wavelet coefficients. An extension of this work reducing the algorithm complexity has recently been proposed [4]. Besides, considering the introduction of a mass preserving constraint, divergence-free wavelets define natural bases for the solutions of the incompressible NavierStokes equations. By the localization of basis functions both in scale and space and by their implicit representation in term of divergence-free motions, these wavelets perfectly describe the vortex structures appearing at various scales of the incompressible flow. These bases have been already used in the simulations of the NavierStokes equations and for the analyses of incompressible fluid flows, with good results [5]. Additionally, expanding the solution on regular wavelet bases enables the easy computation of high-order derivatives and regularization integrals. That have to be mandatory included to cope with the so called aperture problem, which prevents any parametric motion estimation onto too small spatial support.

Gathering these ingredients and constraints, this work aims at proposing a wavelet-based motion estimator that incorporates a high-order smoothing term and a divergence-free condition. Such estimator which extends the approaches of $[4,25]$ is particularly relevant for the estimation of an incompressible fluid flow from two consecutive images. The divergence free constraint, characterizing the physics of incompressible fluid flows, is imposed by directly estimating coefficients of the optical flow projection onto a divergence-free wavelet basis. The methodology takes also benefits from the wavelet continuous formulation to approach or compute exactly high-order regularization integrals, and avoid unstable discrete approximations of the derivatives. In addition, in order to lower the algorithm complexity, efficient quasi-Newton optimization techniques based on wavelets filter banks and the tensor structure of the separable bases are proposed.

The paper is organized as follows. Section 2 gives a brief overview of the concerned problems and highlights the paper contribution. In Section 3 and Section 4, we recall the basic ingredients of optic-flow computation and wavelets basis construction. In Section 5, we perform the biorthogonal wavelet expansion of the motion field which, as we shall see, is necessary to constrain the solution to live in the divergence-free vectorial space. Two approaches for wavelet-based high-order regularization are then introduced in Section 6. Numerical results performed on synthetic and real images of $2 \mathrm{D}$ and $3 \mathrm{D}$ turbulent flows are finally presented and analyzed in Section 7.

\section{Problematic and Contributions}

From a more general point of view, this paper concerns regularized solution $\mathbf{u}^{*}$ of inverse problem written in variational form as

$\mathbf{u}^{*} \in \underset{\mathbf{u} \in E}{\arg \min } F_{d}(\mathbf{u})+\gamma F_{r}(\mathbf{u})$,

where $E$ denotes the space of feasible solutions, $F_{d}$ is a data model adequation term, $F_{r}$ incorporates some regularization constraints on the solution (smoothness, Sobolev norm, differentiation operators norm) and $\gamma>$ 0 . In this paper, the solution of (1) is searched in terms of its wavelet serie discrete coefficients.

Wavelet bases have the great advantage of providing algorithms for the fast computation of derivative operators. In this context, this paper proposes two simple yet efficient approaches to implement high-order differential constraints on the solution appearing in $F_{r}$ : a discrete intuitive approximation method and a continuous and exact approach, which is theoretically more accurate but requires wavelet basis connection coefficients computation. The former has the advantage to yield a linear computational complexity, but is restricted to isotropic regularization operators, whereas the latter can be used to encode any differential smoothing functional. In this paper we apply the method to the case of optical flow estimation and show that this methodology improves state of the art results.

This paper also provides an elegant wavelet-based framework for implementing optimization problem subject to differential constraints. The latter, which are 
often imposed by the physic, are of particular importance in image-based fluid flow or medical images analysis. Among them, well-known constraints are the divergence free condition for incompressible flows or the curl free condition for irrotational flows. The originality of the contribution is to propose an efficient and effective framework incorporating those differential constraints directly in the wavelet basis construction. Such a methodology is not restricted only to motion estimation. It could be as well applied to surface reconstruction, tensor field denoising or other computer vision applications that necessitate the respect of differential constraints.

\section{Basic Principles of Optical Flow Techniques}

This section describes briefly the motion estimation problem, and in particular how it is solved on the canonical basis or on a truncated wavelet basis. The purpose here is not to compare the various approaches proposed so far but rather to introduce general ingredients of wavelets representations and how a motion estimation issue may be handled within such a framework.

\subsection{Problem Formulation}

Given two images denoted $I_{1}(\mathbf{x})$ and $I_{0}(\mathbf{x})$, motion estimation aims as finding a velocity field $\mathbf{u}=\left(u_{1}, u_{2}\right)^{T}$ minimizing the Displaced Frame Difference (DFD) equation $^{1}$ :

$I_{1}(\mathbf{x}+\mathbf{u}(\mathbf{x}))-I_{0}(\mathbf{x})=0$,

hypothesizing a brightness conservation along the trajectory of a 2D image point. Most often, the solution is obtained by minimizing a data adequation term of the form:

$F_{d}(\mathbf{u})=\frac{1}{2} \int_{\mathbb{R}^{2}} \rho\left(I_{1}(\mathbf{x}+\mathbf{u}(\mathbf{x}))-I_{0}(\mathbf{x})\right) d \mathbf{x}$

where $\rho$ may be a robust penalty function. However, for the clarity of the presentation, we will only consider in the following a standard quadratic cost function. Such a functional is not convex because of the non-linearity of the image function $I_{1}$. It is in addition ill-posed as it relies on a scalar constraint for a $2 \mathrm{D}$ vector field unknown. To cope with this strong limitation either a

\footnotetext{
1 In the following, we will restrict ourselves to the study of DFD equation, but the approach remains valid for any other integrated data model. Indeed, for other image modalities, many other brightness evolution models have been proposed in the literature to link the image intensity function to the sought velocity field [15].
}

reduced dimensional parametric representation of the solution or an additional global smoothing constraint is included in the functional. Let us note that in the former case the support of the parametric representation must be sufficiently large in order to circumvent homogeneous photometric areas or step regions with a unique gradient direction for which motion estimation remains ill-posed (the so-called aperture problem).

For small displacements and smooth intensity gradient the Displaced Frame Difference may be replaced by its linear differential counterpart, the so-called optical flow constraint equation:

$\partial_{t} I(\mathbf{x}, t)+\nabla I(\mathbf{x}, t) \cdot \mathbf{u}(\mathbf{x}, t)=0$.

We present hereafter strategies to deal with cases that depart significantly from such a linear assumption (large displacements for instance). This problem occurs particularly within the context of fluid flows, where large velocity fluctuations may be observed under condition of low time-sampling frequency.

\subsection{Estimation on Standard Basis}

An incremental multiresolution strategy stemming from Gauss Newton non-linear least squares is the common method used in optical flow estimation to deal with large displacements [1]. This scheme consists to settle an incremental estimation based on the displaced frame difference linearization around the current estimation. This approach is in general coupled with a multiresolution pyramidal representation of the image data obtained by successive low pass filtering and subsampling. At a given resolution level, the motion field obtained at coarser level is refined considering a linearization of the DFD around the coarse estimate. This scheme is applied within a coarse-to-fine strategy until the finest resolution level. A severe drawback of the method is that the incremental estimation is thus driven at each resolution level on slightly different data (due to low pass filtering and subsampling), resulting theoretically to different minimization problems at each level. This multiresolution construction hence does not generate a family of nested subspaces of solutions.

\section{Standard Wavelet-Based Method}

As opposed to the previous strategy, the wavelet-based optical flow approach first introduced by Wu et al. [25] provides a natural and mathematically consistent multiresolution estimation framework that does not face the theoretical limitations mentioned in Section 3. This 
approach performs the joint estimation of the coefficients of each scalar component of optical flow $\mathbf{u}$ decomposed on a wavelet basis. This decomposition is coherent with the idea used in standard optical flow multiresolution strategy: the inner products with scaling functions are somehow analogous to low-pass filtering operations used for a pyramidal representation. Indeed, wavelet multiresolution analysis is defined by projections onto approximation spaces related to the different scaling functions. The main drawback of the method described in [25] lies in its high computational cost, caused by the necessity to explicitly evaluate the functional Hessian. Recently, a new wavelet-based method that overcomes this computational burden was proposed by Dérian et al. [4]. The main objective in this section is to briefly introduce this method and the principles that underlies the construction of wavelet bases.

\subsection{Wavelet Bases}

To construct wavelet bases, we recall the formalism of multiresolution analysis developed by Mallat [16]. This context leads to the fast wavelet transform algorithm.

By definition [16], a multiresolution analysis of $L^{2}(\mathbb{R})$ is a sequence of closed subspaces denoted $\left\{V_{j}\right\}_{j \in \mathbb{Z}}$ that satisfy:

(i) $V_{j} \subset V_{j+1}, \cap_{j \in \mathbb{Z}} V_{j}=\{0\}, \overline{\cup_{j \in \mathbb{Z}} V_{j}}=L^{2}(\mathbb{R})$.

(ii) For all $f \in L^{2}(\mathbb{R})$ and $\forall j, k \in \mathbb{Z}$ :

$f(x) \in V_{j} \Leftrightarrow f(2 x) \in V_{j+1}$

and

$f(x) \in V_{0} \Leftrightarrow f(x-k) \in V_{0}$.

(iii) There exists a function $\varphi$ such that $\{\varphi(.-k)\}_{k \in \mathbb{Z}}$ is a Riesz basis of $V_{0}$.

The function $\varphi$ is called scaling function. From definition (ii), $\varphi$ satisfies a two-scale (or refinement) relation:

$\varphi(x)=\sum_{k \in \mathbb{Z}} h_{k} \sqrt{2} \varphi(2 x-k), \quad h_{k} \in \mathbb{R}$.

The mask $\left\{h_{k}\right\}_{k \in \mathbb{Z}}$ is called scaling function filter. Moreover, one can prove that the support of $\left\{h_{k}\right\}_{k \in \mathbb{Z}}$ coincides with the support of $\varphi[3]$. The parameter $j$ defines the resolution or the details level. Definition $(i)$ allows to decompose $V_{j+1}$ as:

$V_{j+1}=V_{j} \oplus W_{j}$, where $W_{j}$ denotes the topological complement, which is not unique. The wavelet basis is defined as an unconditional basis of $W_{j}$. Thus, using (ii), the wavelet generator $\psi \in W_{0}$ is defined by its two-scale relation in $V_{1}$ :

$\psi(x)=\sum_{k \in \mathbb{Z}} g_{k} \sqrt{2} \varphi(2 x-k), \quad g_{k} \in \mathbb{R}$.

Similarly, the mask $\left\{g_{k}\right\}_{k \in \mathbb{Z}}$ is called wavelet filter. Setting for $j, k \in \mathbb{Z}, \varphi_{j, k}=2^{j / 2} \varphi\left(2^{j} x-k\right)$ and $\psi_{j, k}=$ $2^{j / 2} \psi\left(2^{j} x-k\right)$, we have:

$V_{j}=\operatorname{span}\left\{\varphi_{j, k}: k \in \mathbb{Z}\right\}, \quad W_{j}=\operatorname{span}\left\{\psi_{j, k}: k \in \mathbb{Z}\right\}$.

Orthogonal wavelet basis concept consists in defining $W_{j}$ as the orthogonal complement of $V_{j}$ :

$V_{j+1}=V_{j} \oplus W_{j}, \quad W_{j}=V_{j+1} \cap\left(V_{j}\right)^{\perp}$.

More generally, one can define $W_{j}$ as:

$V_{j+1}=V_{j} \oplus W_{j}, \quad W_{j}=V_{j+1} \cap\left(\tilde{V}_{j}\right)^{\perp}$.

where $\left\{\tilde{V}_{j}\right\}_{j \in \mathbb{Z}}$ is another multiresolution analysis of $L^{2}(\mathbb{R})$ with scaling $\tilde{\varphi}$ and wavelet $\tilde{\psi}$. In this case, $\left(V_{j}, \tilde{V}_{j}\right)$ is referred as a biorthogonal multiresolution analysis of $L^{2}(\mathbb{R})$. This biorthogonaly property is expressed on the scaling function and wavelet bases by [3]:

$$
\begin{aligned}
& \left\langle\varphi_{j, k}, \tilde{\varphi}_{j, k^{\prime}}\right\rangle=\delta_{k, k^{\prime}}, \quad \forall j, k, k^{\prime} \in \mathbb{Z}, \\
& \left\langle\psi_{j, k}, \tilde{\psi}_{j^{\prime}, k^{\prime}}\right\rangle=\delta_{k, k^{\prime}} \delta_{j, j^{\prime}}, \quad \forall j, j^{\prime}, k, k^{\prime} \in \mathbb{Z}, \\
& \left\langle\varphi_{j, k}, \tilde{\psi}_{j, k^{\prime}}\right\rangle=0, \forall j, k, k^{\prime} \in \mathbb{Z}, \\
& \left\langle\tilde{\varphi}_{j, k}, \psi_{j, k^{\prime}}\right\rangle=0, \quad \forall j, k, k^{\prime} \in \mathbb{Z},
\end{aligned}
$$

where $\delta_{k, k^{\prime}}$ denotes the Kronecker symbol and $<.$, . $>$ the $L^{2}(\mathbb{R})$ inner product:

$\left\langle\varphi_{j, k}, \tilde{\varphi}_{j, k^{\prime}}\right\rangle:=\int_{\mathbb{R}} \varphi_{j, k}(x) \tilde{\varphi}_{j, k^{\prime}}(x) d x$.

One remarks that orthogonal multiresolution analysis is a particular case of the biorthogonal one, where $\varphi=\tilde{\varphi}$ and $\psi=\tilde{\psi}$.

This construction of wavelet bases can be extended easily to $L^{2}\left(\mathbb{R}^{d}\right)(d>1)$ in higher dimension, using tensor product of the one-dimensional wavelet basis [16] Precisely, for any pair of vectors $\mathbf{u}$ and $\mathbf{v}$ of $\mathbb{R}^{d}$, we define the matrix of tensor product $\mathbf{u} \otimes \mathbf{v}$ by:

$[\mathbf{u} \otimes \mathbf{v}]_{i, j}:=u_{i} v_{j}, \quad 1 \leq i, j \leq d$.

Then, using (10) and from a multiresolution analysis $\left\{V_{j}\right\}_{j \in \mathbb{Z}}$ of $L^{2}(\mathbb{R})$, with scaling function $\varphi$, we define the space $V_{j} \otimes V_{j}$ by:

$V_{j} \otimes V_{j}=\operatorname{span}\left\{\varphi_{j, k_{1}} \otimes \varphi_{j, k_{2}}: k_{1}, k_{2} \in \mathbb{Z}\right\}, j \in \mathbb{Z}$, 


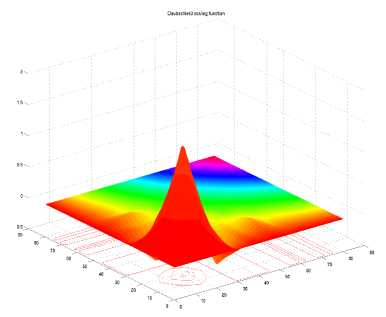

(a) Scaling function $\varphi \otimes \varphi$.

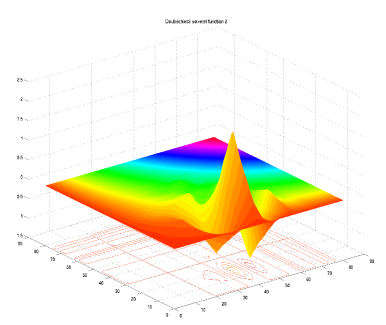

(c) Wavelet $\psi \otimes \varphi$.

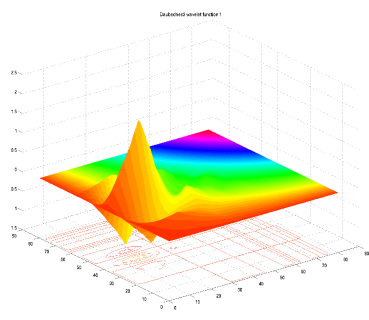

(b) Wavelet $\varphi \otimes \psi$.

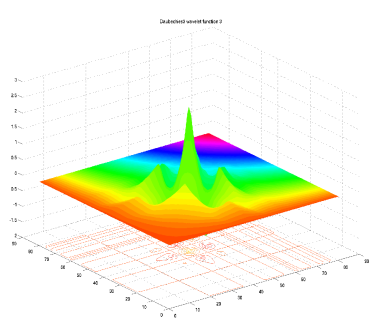

(d) Wavelet $\psi \otimes \psi$.
Fig. 1: Example of 2D scaling function and wavelet generators: case of Daubechies orthogonal wavelet $\psi$ with three vanishing moment.

with

$\varphi_{j, k_{1}} \otimes \varphi_{j, k_{2}}(x, y):=\varphi_{j, k_{1}}(x) \varphi_{j, k_{2}}(y)$.

Thus, the spaces $\left(V_{j} \otimes V_{j}\right)_{j \in \mathbb{Z}}$ constitute a multiresolution analysis of $L^{2}\left(\mathbb{R}^{2}\right)$. The corresponding wavelet spaces are defined using the following two-scale relation:

$$
\begin{aligned}
& V_{j+1} \otimes V_{j+1}=\left(V_{j} \oplus W_{j}\right) \otimes\left(V_{j} \oplus W_{j}\right) \\
& =\left(V_{j} \otimes V_{j}\right) \oplus\left(V_{j} \otimes W_{j}\right) \oplus\left(W_{j} \otimes V_{j}\right) \oplus\left(W_{j} \otimes W_{j}\right) .
\end{aligned}
$$

We have then three types of wavelet generators in $L^{2}\left(\mathbb{R}^{2}\right)$ :

$\varphi \otimes \psi(x, y):=\varphi(x) \psi(y)$ associated to $V_{0} \otimes W_{0}$, $\psi \otimes \varphi(x, y):=\psi(x) \varphi(y)$ associated to $W_{0} \otimes V_{0}$, $\psi \otimes \psi(x, y):=\psi(x) \psi(y)$ associated to $W_{0} \otimes W_{0}$.

This wavelet basis construction is called isotropic construction. It is built from one parameter of dilatation at each scale.

Figure1 shows the plot of isosurfaces of the scaling function generator $\varphi \otimes \varphi$ and the associated three wavelet generators in the isotropic construction, for Daubechies orthogonal wavelet $\psi$ with three vanishing moment [16]. In general, we have $\left(2^{d}-1\right)$ types of wavelet generators in $L^{2}\left(\mathbb{R}^{d}\right)$, see [16] for details.
From relation $(i)$ and equation (5), it is easy to see that the spaces $V_{j}$ verify:

$V_{j}=V_{0} \oplus W_{1} \oplus \cdots \oplus W_{j-1}, \quad j>0$.

Then, instead of having three wavelet basis generators in $L^{2}\left(\mathbb{R}^{2}\right)$, an alternative is to use one wavelet generator with two parameters of translation and dilatation. In this case, it is called anisotropic wavelet basis. This setting consists in decomposing $V_{j} \otimes V_{j}$ as:

$$
\begin{aligned}
& V_{j} \otimes V_{j}=\left(V_{0} \otimes V_{0}\right) \bigoplus_{j_{1}, j_{2}=0}^{j-1}\left(W_{j_{1}} \otimes W_{j_{2}}\right) \\
& =\left(V_{0} \oplus W_{1} \oplus \cdots \oplus W_{j-1}\right) \otimes\left(V_{0} \oplus W_{1} \oplus \cdots \oplus W_{j-1}\right) .
\end{aligned}
$$

The corresponding wavelet basis is thus constituted by:

$\left\{\Psi_{\mathbf{j}, \mathbf{k}}(x, y):=\psi_{j_{1}, k_{1}}(x) \psi_{j_{2}, k_{2}}(y): j_{1}, j_{2}, k_{1}, k_{2} \in \mathbb{Z}\right\}$.

In general, this construction is extended to higher dimension $(d>2)$, with one wavelet generator and $d$ parameters of translation and dilatation. Schematically, the isotropic decomposition of $V_{j} \otimes V_{j}$ is illustrated by Figure 2 and the anisotopic one is illustrated by Figure 3 .

For vector space $\left(L^{2}\left(\mathbb{R}^{d}\right)\right)^{d}$, the construction is done in accordance for each component. For example, a multiresolution analysis of $\left(L^{2}\left(\mathbb{R}^{2}\right)\right)^{2}$ is provided by:

$\mathbf{V}_{j}=\left(V_{j} \otimes V_{j}\right) \times\left(V_{j} \otimes V_{j}\right):=\left(\begin{array}{c}V_{j} \otimes V_{j} \\ V_{j} \otimes V_{j}\end{array}\right)$.

\subsection{Fast Wavelet Transform}

The multi-scale analysis of $f \in L^{2}(\mathbb{R})$ consists in its decomposition in a coarse approximation $\mathcal{P}_{0}(f) \in V_{0}$ and the sum of details $\mathcal{Q}_{j}(f) \in W_{j}$ :

$f=\mathcal{P}_{0}(f)+\sum_{j \geq 0} \mathcal{Q}_{j}(f), \quad \mathcal{Q}_{j}(f)=\mathcal{P}_{j+1}(f)-\mathcal{P}_{j}(f)$.

For biorthogonal multiresolution analyses, these projections are defined by:

$\mathcal{P}_{j}(f):=\sum_{k \in \mathbb{Z}} c_{j, k} \varphi_{j, k} \quad$ and $\quad c_{j, k}=\left\langle\tilde{\varphi}_{j, k}, f\right\rangle$,

and

$\mathcal{Q}_{j}(f):=\sum_{k \in \mathbb{Z}} d_{j, k} \psi_{j, k} \quad$ and $\quad d_{j, k}=\left\langle\tilde{\psi}_{j, k}, f\right\rangle$.

The fast wavelet transform (FWT) algorithm allows to compute in practice the sequences of scaling function 
coefficients $\left\{c_{j, k}=\left\langle f, \tilde{\varphi}_{j, k}\right\rangle, k \in \mathbb{Z}\right\}$ and wavelet coefficients $\left\{d_{j, k}=\left\langle f, \tilde{\psi}_{j, k}\right\rangle, k \in \mathbb{Z}\right\}$ from $\left\{c_{j+1, k}, k \in \mathbb{Z}\right\}$. To this end, ones uses the following two-scale relations [16]:

$c_{j, k}=\sum_{l \in \mathbb{Z}} \tilde{h}_{l-2 k} c_{j+1, l} \quad$ and $\quad d_{j, k}=\sum_{l \in \mathbb{Z}} \tilde{g}_{l-2 k} c_{j+1, l}$.

This algorithm can be summarized schematically by:

$$
\begin{array}{cccccccccc}
c_{J} & \rightarrow & c_{J-1} & \rightarrow & c_{J-2} & \rightarrow & \ldots & c_{1} & \rightarrow & c_{0} \\
& \searrow & & \searrow & & \searrow & & & \searrow & \\
& & d_{J-1} & & d_{J-2} & & \ldots & d_{1} & & d_{0}
\end{array}
$$

The inverse transform (IWT) is computed using the following reconstruction relation [16]:

$c_{j+1, k}=\sum_{l \in \mathbb{Z}} h_{k-2 l} c_{j, l}+\sum_{l \in \mathbb{Z}} g_{k-2 l} d_{j, l}$,

which algorithm is also schematically pictured as:

$$
\begin{array}{cccccccccc}
c_{0} & \rightarrow & c_{1} & \rightarrow & c_{2} & \rightarrow & \ldots & c_{J-1} & \rightarrow & c_{J} \\
& \nearrow & & \nearrow & & \nearrow & & & \nearrow & \\
d_{0} & & d_{1} & & d_{2} & & \ldots & d_{J-1} & &
\end{array}
$$

The theoretical complexity of the FWT and IWT algorithms is about $O\left(N=2^{J}\right)$, see [16]. In higher dimension $(d>1)$, for the sake of simplicity and easy implementation, we will use the anisotropic setting: to compute the fast wavelet transform in this case, it suffices to apply the one dimensional transform along each direction.

\subsection{Standard Wavelet-Based Flow Estimation}

Let us introduce some shorthand notations: the 2D scaling function basis of $V_{j} \otimes V_{j}$ is denoted $\Phi_{j}$, and $\Psi_{\mathbf{j}}$, $|\mathbf{j}| \leq j$ the corresponding anisotropic wavelets basis. Similarly for the dual space $\tilde{V}_{j} \otimes \tilde{V}_{j}$ with $\tilde{\Phi}_{j}$ and $\tilde{\Psi}_{\mathbf{j}}$. For $f \in L^{2}\left(\mathbb{R}^{2}\right)$, the associated multi-scale projectors are denoted:

$\mathcal{P}_{j}(f)=\left\langle\tilde{\Phi}_{j}, f\right\rangle \Phi_{j}, \quad \mathcal{Q}_{\mathbf{j}}(f)=\left\langle\tilde{\Psi}_{\mathbf{j}}, f\right\rangle \Psi_{\mathbf{j}}, \quad|\mathbf{j}| \leq j$ and we make the same conventions in $\left(L^{2}\left(\mathbb{R}^{2}\right)\right)^{2}$ with: $\mathbf{P}_{j}(\mathbf{f})=\left\langle\tilde{\boldsymbol{\Phi}}_{j}, \mathbf{f}\right\rangle \boldsymbol{\Phi}_{j}, \quad \mathbf{Q}_{\mathbf{j}}(\mathbf{f})=\left\langle\tilde{\boldsymbol{\Psi}}_{\mathbf{j}}, \mathbf{f}\right\rangle \boldsymbol{\Psi}_{\mathbf{j}}, \quad|\mathbf{j}| \leq j$.

With the previous shorthand notations, the method of [4] consists in searching, at each scale $j$, motion field $\mathbf{u}_{j}$ represented on a wavelet basis at scale $\mathrm{j}$ :

$\mathbf{u}_{j}(\mathbf{x})=\mathbf{d}_{j} \boldsymbol{\Psi}_{\mathbf{j}}(\mathbf{x}), \quad \mathbf{d}_{j}=\left\langle\tilde{\Psi}_{\mathbf{j}}, \mathbf{u}\right\rangle, \quad|\mathbf{j}| \leq j$,

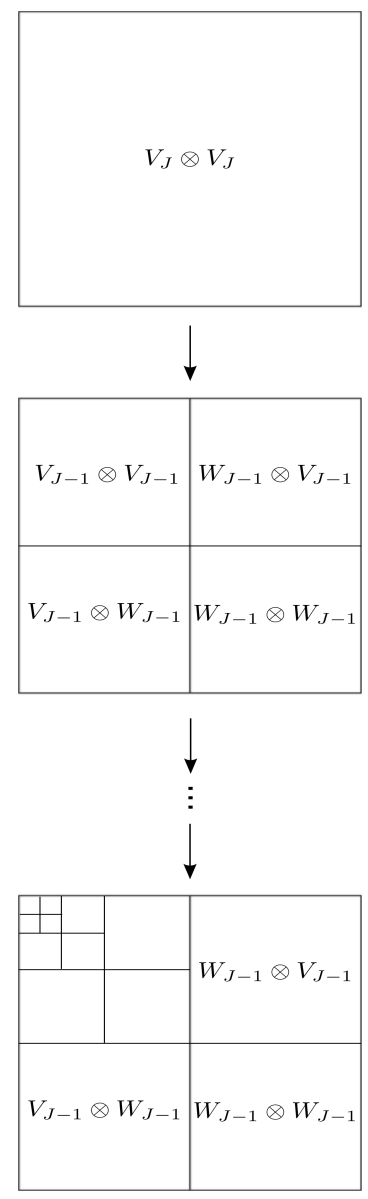

Fig. 2: 2D Isotropic Fast Wavelet Transform scheme.

where $\boldsymbol{\Psi}_{\mathbf{j}}$ is the selected $2 \mathrm{D}$ wavelet basis and $\mathbf{d}_{j}$ the vector of unknown coefficients that have to be estimated to represent the sought motion components. Incorporating (17) in the (DFD) equation and setting $I_{1}\left(\mathbf{x}, \mathbf{d}_{j}\right)=I_{1}\left(\mathbf{x}+\mathbf{d}_{j} \Psi_{\mathbf{j}}(\mathbf{x})\right)$, the gradient of $F_{d}$ according to the unknown coefficients reads:

$\nabla F_{d}\left(\mathbf{d}_{j}\right)=\int\left[I_{1}\left(\mathbf{x}, \mathbf{d}_{j}\right)-I_{0}(\mathbf{x})\right] \nabla I_{1}\left(\mathbf{x}, \mathbf{d}_{j}\right) \cdot \Psi_{\mathbf{j}}(\mathbf{x}) d \mathbf{x}$.

As a consequence, components of the gradient $\nabla F_{d}\left(\mathbf{d}_{j}\right)$ of the functional are simply given by the coefficients of the wavelet decomposition of the two components of gradient:

$\left[I_{1}\left(\mathbf{x}, \mathbf{d}_{j}\right)-I_{0}(\mathbf{x})\right] \nabla I_{1}\left(\mathbf{x}, \mathbf{d}_{j}\right)$,

on the considered dual wavelet basis. For a given motion field, they can be easily computed using a 2D fast wavelet transform [16], with the filter bank associated to $\boldsymbol{\Psi}_{\mathbf{j}}$. This proposed coarse-to-fine estimation strategy enables to capture large displacements: at large scales, the decomposition of (18) is obtained by convolutions with the atoms of the wavelet basis having the 


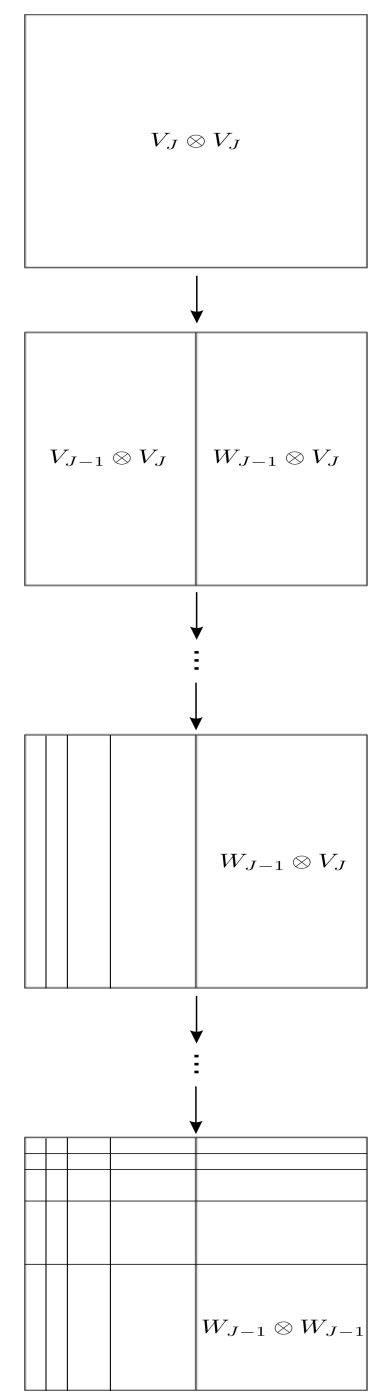

Fig. 3: 2D Anisotropic Fast Wavelet Transform scheme.

largest support. A gradient descent method is used then to minimize efficiently the functional $F_{d}$. The aperture problem can be here jointly addressed by reducing the problem dimension with a simple basis truncation: coefficients associated to smallest scales are not estimated, finest wavelet supports is large enough to cope with homogeneous areas or gradients unique direction (linear step in-between two homogeneous regions). Obviously, the choice of this finest scale is not an easy issue in general. It should be clearly handled with some adaptive strategy. The other solution consists in adjoining an additional smooth constraint to the functional. This will be detailed in Section 6 .

Let us suppose images of size $2^{J} \times 2^{J}$. To estimate the motion at scale $j<J$, starting with an initial co- efficients $\mathbf{d}_{j}$ of length $2 \times 2^{j} \times 2^{j}$, one step of the optimization procedure requires to:

1. Compute $\mathbf{u}_{j}$ at fine grid points by extrapolation.

2. Compute $I_{1}\left(\mathbf{x}+\mathbf{u}_{j}\right)$ using interpolation.

3. Evaluate $F_{d}$ on $\mathbf{u}_{j}$ using quadrature formula.

4. Compute $\nabla I_{1}\left(\mathbf{x}+\mathbf{u}_{j}\right)$ with finite difference method.

5. Compute the gradient $\nabla F_{d}\left(\mathbf{d}_{j}\right)$ to implement a gradient descent method.

Wavelet bases are used only in Step 1 and Step 5. Precisely, Step 1 corresponds to an inverse fast wavelet transform on coefficients $\mathbf{d}_{j}$ and Step 5 corresponds to the fast wavelet transform of $\left[I_{1}\left(\mathbf{x}+\mathbf{u}_{j}\right)-I_{0}(\mathbf{x})\right] \nabla I_{1}\left(\mathbf{x}+\mathbf{u}_{j}\right)$. Thus, the theoretical complexity of these steps is $O(N)$, with $N=2^{J} \times 2^{J}$.

Additionally to the necessarily introduction of regularization term to reach the finest scale levels it is also in some situation mandatory to consider additional physical constraints in the estimation process. This is the case when one aims for instance at estimating volume preserving or diffeomorphic transformation. Such constraints are generally imposed through differential constraints. Wavelet bases specifically designed to satisfy theses relations may be advantageous. In the following, we show how the estimation of a volume preserving transformation may be taken into account in the wavelet design.

\section{Divergence-Free Wavelet-Based Method}

Vector field analysis is ubiquitous in almost all the scientific domains. The Helmholtz decomposition theorem, sometimes referred as the fundamental theorem of vector calculus, states that any sufficiently smooth vector field $\mathbf{u} \in\left(L^{2}\left(\mathbb{R}^{d}\right)\right)^{d}$ can be decomposed uniquely in the form:

$\mathbf{u}=\mathbf{u}^{\text {div }}+\mathbf{u}^{\text {curl }}$,

with

$\operatorname{div}\left(\mathbf{u}^{\operatorname{div}}\right)=0 \quad$ and $\quad \operatorname{curl}\left(\mathbf{u}^{\text {curl }}\right)=0$.

Constraints like (20) appear in many computer vision problems (for example in medical image registration [12], image restoration [21], optical flow estimation [19, 20], or in engineering problems (incompressible turbulence flow simulation). 
In the case of optical flow estimation, since the cost function defined in (3) is under constrained, to make its minimization mathematically well-posed, it is important to introduce some prior knowledge on the kind of typical flow one expects to estimate. The estimation of diffeomorphic transformation [21] or of mass preserving fluid flow $[19,20]$ leads to consider divergence-free motion field. To achieve this, the minimization is coupled with a resolution of incompressible Stokes system of partial differential equations, which requires discretization on staggered grids to get stable solution [26].

Wavelet bases can be designed to implicitly represent divergence-free or curl-free motions [5]. Without loss of generality, we will focus on the divergencefree constraint. The curl-free constraint or the two constraints both can be treated similarly. Then, the purpose of this section is to detail the construction of anisotropic divergence-free wavelet bases in $\left(L^{2}\left(\mathbb{R}^{2}\right)\right)^{2}$ that are used to define a new divergence-free wavelet based method for optical flow estimation.

\subsection{Divergence-Free Wavelet Basis}

The objective here is to build an anisotropic wavelet basis for the divergence-free functions space $\mathcal{H}_{\text {div }}\left(\mathbb{R}^{2}\right)$ defined by:

$\mathcal{H}_{\text {div }}\left(\mathbb{R}^{2}\right)=\left\{\mathbf{u} \in\left(L^{2}\left(\mathbb{R}^{2}\right)\right)^{2}: \nabla \cdot \mathbf{u}=0\right\}$.

Alternatively, the space $\mathcal{H}_{\text {div }}\left(\mathbb{R}^{2}\right)$ can also be seen as the curl $^{2}$ vector potential space $[6]^{3}$ :

$\mathcal{H}_{\text {div }}\left(\mathbb{R}^{2}\right)=\left\{\mathbf{u}=\operatorname{curl}(\chi): \chi \in H^{1}\left(\mathbb{R}^{2}\right)\right\}$.

Let us recall the following formal identities, valid for $d=2$ and $d=3$ :

$\operatorname{div}(\operatorname{curl})=0 \quad$ and $\quad \operatorname{curl}(\nabla)=0$.

From (22), to construct a multiresolution analysis of $\mathcal{H}_{\text {div }}\left(\mathbb{R}^{2}\right)$, it is natural to take the curl of a "regular" scalar multiresolution analysis of $H^{1}\left(\mathbb{R}^{2}\right)$.

For practical reasons, the effective divergence-free wavelet bases have to be compactly supported and also to give rise to a fast divergence-free wavelet transform

${ }^{2}$ For $d=2$, we define $\operatorname{curl}(\chi):=\left(\partial_{y} \chi,-\partial_{x} \chi\right)$.

${ }^{3} H^{1}\left(\mathbb{R}^{d}\right)$ denotes the classical Sobolev space:

$$
\|f\|_{H^{1}\left(\mathbb{R}^{d}\right)}^{2}=\|f\|_{L^{2}\left(\mathbb{R}^{d}\right)}^{2}+\|\nabla f\|_{L^{2}\left(\mathbb{R}^{d}\right)}^{2}
$$

algorithm. Such wavelet bases exist and can be fortunately easily constructed. They have been introduced first by Lemarié-Rieusset [14].

Before further introducing this construction, we first give some technical precisions. Since the curl operator introduces derivation operators, it is important to answer to the following questions: what is the derivative of a scaling function? What is the derivative of a wavelet? Does differentiation preserve the $L^{2}(\mathbb{R})$ orthogonality property of a wavelet basis?

Using integration by part, to answer the last question we have:

$\int_{\mathbb{R}} \varphi_{k}^{\prime}(x) \varphi_{\ell}^{\prime}(x) d x=-\int_{\mathbb{R}} \varphi_{k}(x) \varphi_{\ell}^{\prime \prime}(x) d x$.

Thus, if $\left\{\varphi_{j, k}: k \in \mathbb{Z}\right\}$ is an orthogonal basis, this property is preserved by differentiation if and only if $\varphi=-\varphi^{\prime \prime}$ : which is for instance true for sinus or cosinus basis.

The answers to the first two questions are supplied by the following results proved by Lemarié-Rieusset [13]:

\section{Proposition 1}

Let $\left(\varphi^{1}, \tilde{\varphi}^{1}\right)$ be a pair of biorthogonal scaling functions associated to biorthogonal wavelets $\left(\psi^{1}, \tilde{\psi}^{1}\right)$, with $\varphi^{1} \in$ $\mathcal{C}^{1+\epsilon}(\mathbb{R}), \epsilon>0$. Then there exists another biorthogonal scaling functions $\left(\varphi^{0}, \tilde{\varphi}^{0}\right)$ and biorthogonal wavelets $\left(\psi^{0}, \tilde{\psi}^{0}\right)$, satisfying:

$\frac{d}{d x} \varphi^{1}(x)=\varphi^{0}(x)-\varphi^{0}(x-1)$,

and

$\frac{d}{d x} \tilde{\varphi}^{0}(x)=\tilde{\varphi}^{1}(x+1)-\tilde{\varphi}^{1}(x)$.

The associated wavelets verify:

$\psi^{1}(x)=4 \int_{-\infty}^{x} \psi^{0} \quad$ and $\quad \tilde{\psi}^{0}(x)=-4 \int_{-\infty}^{x} \tilde{\psi}^{1}$.

Hence, according to Proposition 1, the derivative of a scaling function is expressed as a finite difference on neighboring hoods of another scaling function and the derivative of a wavelet is another wavelet. Figure 4 shows the plot of an example of these scaling functions and wavelets satisfying Proposition 1 and related by differentiation and integration.

Let $\left(V_{j}^{1}\right)_{j \in \mathbb{Z}}$ and $\left(V_{j}^{0}\right)_{j \in \mathbb{Z}}$ be one-dimensional multiresolution analyses of $L^{2}(\mathbb{R})$ provided by $\varphi^{1}$ and $\varphi^{0}$ respectively, with $\varphi^{1}$ and $\varphi^{0}$ defined by Proposition 1 . 
Since we use tensor product construction in higher dimension, each sequence of space, namely:

$V_{j}^{1} \otimes V_{j}^{0}=\operatorname{span}\left\{\varphi_{j, k_{1}}^{1}(x) \varphi_{j, k_{2}}^{0}(y), k_{1}, k_{2} \in \mathbb{Z}\right\}, \quad j \in \mathbb{Z}$,

or

$V_{j}^{0} \otimes V_{j}^{1}=\operatorname{span}\left\{\varphi_{j, k_{1}}^{0}(x) \varphi_{j, k_{2}}^{1}(y), k_{1}, k_{2} \in \mathbb{Z}\right\}, \quad j \in \mathbb{Z}$,

form a multiresolution analysis of $L^{2}\left(\mathbb{R}^{2}\right)$. Moreover, to compute the fast wavelet decomposition, in the multiresolution generated by $V_{j}^{1} \otimes V_{j}^{0}$, it suffices to use the filters of $\left(\tilde{\varphi}^{1}, \tilde{\psi}^{1}\right)$ in the $x$ direction and those of $\left(\tilde{\varphi}^{0}, \tilde{\psi}^{0}\right)$ in the $y$ direction. The reconstruction is done with the filters of $\left(\varphi^{1}, \psi^{1}\right)$ and $\left(\varphi^{0}, \psi^{0}\right)$ respectively.

From relations (23) and (24), one can derive two interesting properties of biorthogonal multiresolution analyses $\left(V_{j}^{1}, \tilde{V}_{j}^{1}\right)$ and $\left(V_{j}^{0}, \tilde{V}_{j}^{0}\right)$ :

\section{Corollary 1 [13]}

Let $\left(V_{j}^{1}, \tilde{V}_{j}^{1}\right)$ and $\left(V_{j}^{0}, \tilde{V}_{j}^{0}\right)$ be two BMRAs of $L^{2}(\mathbb{R})$ that satisfy Proposition 1, then we have:

(a) $\frac{d}{d x} V_{j}^{1}=V_{j}^{0}, \quad \tilde{V}_{j}^{0}=\int_{-\infty}^{x} \tilde{V}_{j}^{1}$,

and

(b) $\frac{d}{d x} \mathcal{P}_{j}^{1}(f)=\mathcal{P}_{j}^{0}\left(\frac{d}{d x} f\right), \quad \frac{d}{d x} \tilde{\mathcal{P}}_{j}^{0}(f)=\tilde{\mathcal{P}}_{j}^{1}\left(\frac{d}{d x} f\right)$,

for $f \in H^{1}(\mathbb{R})$.

The interest of relations (26) and (27) appears in the numerical implementation of fast divergence-free wavelet transform. These relations allow to build a multiresolution analysis of $\left(L^{2}\left(\mathbb{R}^{2}\right)\right)^{2}$ that preserves the divergence-free property [14].

As stated in $(22)$, the space $\mathcal{H}_{\text {div }}\left(\mathbb{R}^{2}\right)$ corresponds to the curl of $H^{1}\left(\mathbb{R}^{2}\right)$ scalar potential. Then, taking the curl of any multiresolution analysis of $H^{1}\left(\mathbb{R}^{2}\right)$ will provide a multiresolution analysis of $\mathcal{H}_{\text {div }}\left(\mathbb{R}^{2}\right)$. However, let us consider a "regular" scalar multiresolution analysis of $H^{1}\left(\mathbb{R}^{2}\right)$ generated by spaces $V_{j}^{a} \otimes V_{j}^{b}$, with $V_{j}^{a} \neq V_{j}^{b}$. Taking the curl of a such multiresolution analysis, we get:

$\operatorname{curl}\left[V_{j}^{a} \otimes V_{j}^{b}\right]=\mid \begin{gathered}V_{j}^{a} \otimes\left(V_{j}^{b}\right)^{\prime} \\ -\left(V_{j}^{a}\right)^{\prime} \otimes V_{j}^{b}\end{gathered}$

Then, to deal with the divergence-free wavelets contained in $\operatorname{curl}\left[V_{j}^{a} \otimes V_{j}^{b}\right]$, we have to manipulate four

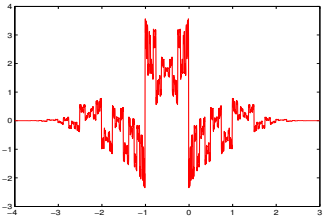

(a) Scaling function $\tilde{\varphi}^{1}$.

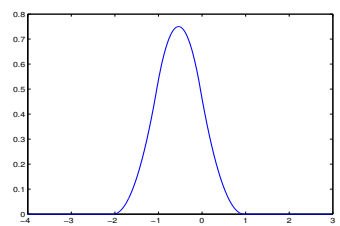

(c) Scaling function $\varphi^{1}$.

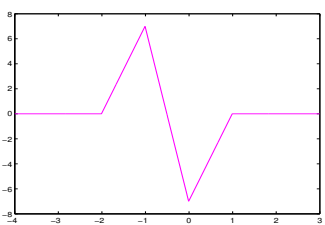

(e) Function $\frac{d}{d x} \varphi^{1}$.

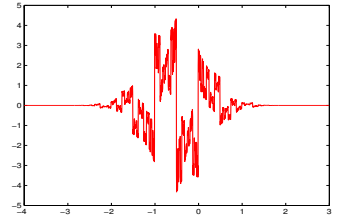

(b) Wavelet $\tilde{\psi}^{1}$.

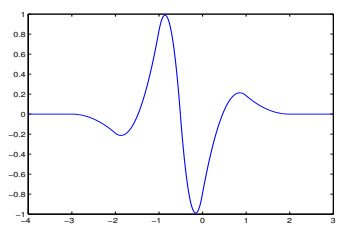

(d) Wavelet $\psi^{1}$.

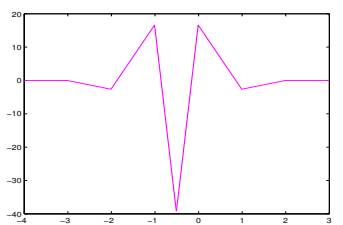

(f) Wavelet $\frac{d}{d x} \psi^{1}=4 \psi^{0}$.
Fig. 4: Example of biorthogonal generators and primal derivatives: case of B-Spline generators $\left(\varphi^{1}, \tilde{\varphi}^{1}\right)$ with 3 vanishing moments.

different types of biorthogonal wavelet filter banks associated respectively to the one-dimensional BMRAs that appear in (28): $V_{j}^{a},\left(V_{j}^{a}\right)^{\prime}, V_{j}^{b}$ and $\left(V_{j}^{b}\right)^{\prime}$. To overcome this problem, the two-dimensional scalar multiresolution analysis that we will consider is generated by spaces $V_{j}^{1} \otimes V_{j}^{1}$.

Using Lemarié-Rieusset's results (23) and (24), one can easily prove that:

$\operatorname{curl}\left(V_{j}^{1} \otimes V_{j}^{1}\right) \subset\left(V_{j}^{1} \otimes V_{j}^{0}\right) \times\left(V_{j}^{0} \otimes V_{j}^{1}\right)=\mathbf{V}_{j}$

Moreover, the multiresolution analysis of $\left(L^{2}\left(\mathbb{R}^{2}\right)\right)^{2}$ provided by spaces $\mathbf{V}_{j}=\left(V_{j}^{1} \otimes V_{j}^{0}\right) \times\left(V_{j}^{0} \otimes V_{j}^{1}\right)$ preserves the divergence-free condition:

$\forall \mathbf{u} \in \mathcal{H}_{\text {div }}\left(\mathbb{R}^{2}\right), \quad \operatorname{div}\left(\mathbf{P}_{j}(\mathbf{u})\right)=P_{j}^{0}(\operatorname{div}(\mathbf{u}))=0$,

where

$\mathbf{P}_{j}=\left(\mathcal{P}_{j}^{1} \otimes \mathcal{P}_{j}^{0}\right) \times\left(\mathcal{P}_{j}^{0} \otimes \mathcal{P}_{j}^{1}\right)$,

and

$P_{j}^{0}=\mathcal{P}_{j}^{0} \otimes \mathcal{P}_{j}^{0}$. 
Accordingly, the divergence-free scaling functions spaces are defined by:

$\mathbb{V}_{j}^{d i v}=\operatorname{span}<\Phi_{j, \mathbf{k}}^{d i v} ; \mathbf{k} \in \mathbb{Z}^{2}, j \in \mathbb{Z}>$,

where

$\boldsymbol{\Phi}_{j, \mathbf{k}}^{d i v}=\operatorname{curl}\left[\varphi_{j, k_{1}}^{1} \otimes \varphi_{j, k_{2}}^{1}\right]=\mid \begin{gathered}\varphi_{j, k_{1}}^{1} \otimes\left(\varphi_{j, k_{2}}^{1}\right)^{\prime} \\ -\left(\varphi_{j, k_{1}}^{1}\right)^{\prime} \otimes \varphi_{j, k_{2}}^{1}\end{gathered}$

Similarly, for $\mathbf{j}, \mathbf{k} \in \mathbb{Z}^{2}$, the associated anisotropic divergence-free wavelet spaces are defined by:

$\mathbb{W}_{\mathbf{j}, \mathbf{k}}^{d i v}=\operatorname{span}<\boldsymbol{\Psi}_{\mathbf{j}, \mathbf{k}}^{d i v} ; \mathbf{j}, \mathbf{k} \in \mathbb{Z}^{2}>$

with

$\mathbf{\Psi}_{\mathbf{j}, \mathbf{k}}^{d i v}=\operatorname{curl}\left[\psi_{j_{1}, k_{1}}^{1} \otimes \psi_{j_{1}, k_{2}}^{1}\right]=\mid \begin{gathered}\psi_{j_{1}, k_{1}}^{1} \otimes\left(\psi_{j_{2}, k_{2}}^{1}\right)^{\prime} \\ -\left(\psi_{j_{1}, k_{1}}^{1}\right)^{\prime} \otimes \psi_{j_{2}, k_{2}}^{1}\end{gathered}$

Differentiating the relations of equation (25), we get:

$\left(\psi^{1}(x)\right)^{\prime}=4 \psi^{0}(x) \quad$ and $\quad\left(\tilde{\psi}^{0}(x)\right)^{\prime}=-4 \tilde{\psi}^{1}(x)$,

thus,

$\boldsymbol{\Psi}_{\mathbf{j}, \mathbf{k}}^{d i v}=\mid \begin{gathered}2^{j_{2}+2} \psi_{j_{1}, k_{1}}^{1} \otimes \psi_{j_{2}, k_{2}}^{0} \\ -2^{j_{1}+2} \psi_{j_{1}, k_{1}}^{0} \otimes \psi_{j_{2}, k_{2}}^{1}\end{gathered}$

These wavelets are biorthogonal [5] and every vector field $\mathbf{u} \in \mathcal{H}_{\text {div }}\left(\mathbb{R}^{2}\right)$ can be decomposed uniquely as:

$\mathbf{u}=\sum_{\mathbf{j}, \mathbf{k} \in \mathbb{Z}^{2}}\left\langle\mathbf{u}, \tilde{\mathbf{\Psi}}_{\mathbf{j}, \mathbf{k}}^{d i v}\right\rangle \mathbf{\Psi}_{\mathbf{j}, \mathbf{k}}^{d i v}=\sum_{\mathbf{j}, \mathbf{k} \in \mathbb{Z}^{2}} \mathbf{d}_{\mathbf{j}, \mathbf{k}}^{d i v} \Psi_{\mathbf{j}, \mathbf{k}}^{d i v}$

where $\langle.,$.$\rangle denotes the \left(L^{2}\left(\mathbb{R}^{2}\right)\right)^{2}$-inner product between two vector functions. On Fig. 5, we plot an example of vector fields and corresponding vorticities of the divergence-free scaling function generator and wavelet generator, constructed from Coifflet $\varphi^{1}$ and $\psi^{1}$ with ten vanishing moments.

\section{Remark 1}

In practice, as mentioned earlier two types of divergence-free wavelet can be built: the isotropic construction [14] and the anisotropic one [5]. The isotropic divergence-free wavelet construction on $\mathbb{R}^{d}$ uses $(d-$ $1)\left(2^{d}-1\right)$ types of wavelet generators with one parameter of dilatation and $d$ parameters of translation, while the anisotropic one uses only $(d-1)$ types of wavelet generators with $d$ parameters of dilation and translation. Thus, for $d=2$, we have one divergencefree wavelet generator in the anisotropic construction.

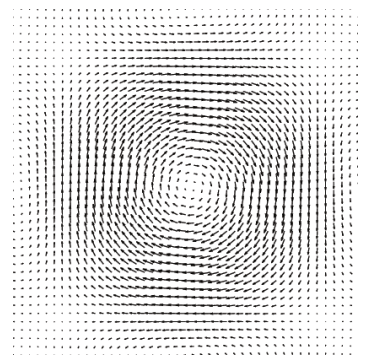

(a) Generator curl $\left(\varphi^{1} \otimes\right.$ $\left.\varphi^{1}\right)$

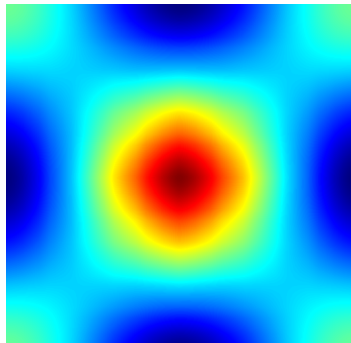

(c) Vorticity of curl $\left(\varphi^{1} \otimes\right.$ $\left.\varphi^{1}\right)$

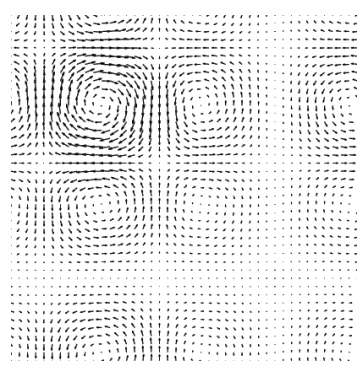

(b) Generator curl $\left(\psi^{1} \otimes\right.$ $\left.\psi^{1}\right)$

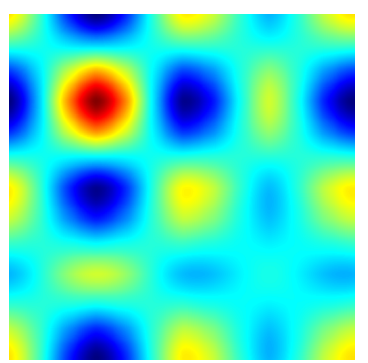

(d) Vorticity of curl $\left(\psi^{1} \otimes\right.$ $\left.\psi^{1}\right)$
Fig. 5: Vector fields and vorticity of divergence-free scaling functions and wavelets generators constructed from Coifflet $\varphi^{1}$ and $\psi^{1}$ with ten vanishing moments.

\subsection{Fast Divergence-Free Wavelet Transform}

By construction, we have seen that the vector spaces $\mathbf{V}_{j}=\left(V_{j}^{1} \otimes V_{j}^{0}\right) \times\left(V_{j}^{0} \otimes V_{j}^{1}\right)$ constitute a multiresolution analysis of $\left(L^{2}\left(\mathbb{R}^{2}\right)\right)^{2}$ and this multiresolution analysis preserves the divergence-free constraint. From a standard anisotropic vector wavelet decomposition associated to $\mathbf{V}_{j}$, the objective of this section is to describe how to compute in practice the anisotropic divergence-free wavelet decomposition of any $\mathbf{u} \in \mathcal{H}_{d i v}\left(\mathbb{R}^{2}\right)$.

The standard anisotropic vector wavelet associated to $\mathbf{V}_{j}$ are:

$\mathbf{\Psi}_{\mathbf{j}, \mathbf{k}}^{1}=\left|\begin{array}{c}\psi_{j_{1}, k_{1}}^{1} \otimes \psi_{j_{2}, k_{2}}^{0}, \\ 0\end{array}, \quad \mathbf{\Psi}_{\mathbf{j}, \mathbf{k}}^{2}=\right| \begin{gathered}0 \\ \psi_{j_{1}, k_{1}}^{0} \otimes \psi_{j_{2}, k_{2}}^{1}\end{gathered}$.

Since $\mathbf{u}=\left(u_{1}, u_{2}\right)$ belongs to $\left(L^{2}\left(\mathbb{R}^{2}\right)\right)^{2}$ and $\left(\Psi_{\mathbf{j}, \mathbf{k}}^{1}, \Psi_{\mathbf{j}, \mathbf{k}}^{2}\right)_{\mathbf{j}, \mathbf{k} \in \mathbb{Z}^{2}}$ is a wavelet basis of $\left(L^{2}\left(\mathbb{R}^{2}\right)\right)^{2}$, we get:

$\mathbf{u}=\sum_{\mathbf{j}, \mathbf{k} \in \mathbb{Z}^{2}} \mathbf{d}_{\mathbf{j}, \mathbf{k}}^{1} \Psi_{\mathbf{j}, \mathbf{k}}^{1}+\sum_{\mathbf{j}, \mathbf{k} \in \mathbb{Z}^{2}} \mathbf{d}_{\mathbf{j}, \mathbf{k}}^{2} \Psi_{\mathbf{j}, \mathbf{k}}^{2}$.

Through an easy calculation, by identification one can show that:

$u_{1}=\sum_{\mathbf{j}, \mathbf{k} \in \mathbb{Z}^{2}} \mathbf{d}_{\mathbf{j}, \mathbf{k}}^{1} \psi_{j_{1}, k_{1}}^{1} \otimes \psi_{j_{2}, k_{2}}^{0}$, 
and

$u_{2}=\sum_{\mathbf{j}, \mathbf{k} \in \mathbb{Z}^{2}} \mathbf{d}_{\mathbf{j}, \mathbf{k}}^{2} \psi_{j_{1}, k_{1}}^{0} \otimes \psi_{j_{2}, k_{2}}^{1}$.

Following [5] and using the relation:

$\boldsymbol{\Psi}_{\mathbf{j}, \mathbf{k}}^{d i v}=2^{j_{2}+2} \boldsymbol{\Psi}_{\mathbf{j}, \mathbf{k}}^{1}-2^{j_{1}+2} \boldsymbol{\Psi}_{\mathbf{j}, \mathbf{k}}^{2}$

we find:

$\mathbf{d}_{\mathbf{j}, \mathbf{k}}^{\text {div }}=\frac{2^{j_{2}+2}}{4^{j_{1}+2}+4^{j_{2}+2}} \mathbf{d}_{\mathbf{j}, \mathbf{k}}^{1}-\frac{2^{j_{1}+2}}{4^{j_{1}+2}+4^{j_{2}+2}} \mathbf{d}_{\mathbf{j}, \mathbf{k}}^{2}$,

and

$\mathbf{d}_{\mathbf{j}, \mathbf{k}}^{1}=2^{j_{2}+2} \mathbf{d}_{\mathbf{j}, \mathbf{k}}^{d i v}, \quad \mathbf{d}_{\mathbf{j}, \mathbf{k}}^{2}=-2^{j_{1}+2} \mathbf{d}_{\mathbf{j}, \mathbf{k}}^{d i v}$.

Therefore, decomposition and reconstruction associated to divergence-free wavelets is simply performed using scalar wavelet filter banks. Finally, the algorithm is of low complexity and its structure remains identical to the scalar case.

The algorithm is summarized then as follows. Starting with $\mathbf{u}=\left(u_{1}, u_{2}\right)$, to get the divergence-free wavelet coefficients $\mathbf{d}_{\mathbf{j}, \mathbf{k}}^{\text {div }}$, do:

1a. Compute $\mathbf{d}_{\mathbf{j}, \mathbf{k}}^{1}$ associated to $u_{1}$ in $V_{j}^{1} \otimes V_{j}^{0}$.

2a. Compute $\mathbf{d}_{\mathbf{j}, \mathbf{k}}^{2}$ associated to $u_{2}$ in $V_{j}^{0} \otimes V_{j}^{1}$.

3a. Compute $\mathbf{d}_{\mathbf{j}, \mathbf{k}}^{\text {div }}$ from $\mathbf{d}_{\mathbf{j}, \mathbf{k}}^{1}$ and $\mathbf{d}_{\mathbf{j}, \mathbf{k}}^{2}$ using (36).

For the reconstruction, do:

1b. Compute $\mathbf{d}_{\mathbf{j}, \mathbf{k}}^{1}$ and $\mathbf{d}_{\mathbf{j}, \mathbf{k}}^{2}$ from $\mathbf{d}_{\mathbf{j}, \mathbf{k}}^{\text {div }}$ using (37).

2b. Compute $u_{1}$ from $\mathbf{d}_{\mathbf{j}, \mathbf{k}}^{1}$ in $V_{j}^{1} \otimes V_{j}^{0}$.

3b. Compute $u_{2}$ from $\mathbf{d}_{\mathbf{j}, \mathbf{k}}^{2}$ in $V_{j}^{0} \otimes V_{j}^{1}$.

Steps $1 a$ and Step $2 a$ correspond to a two dimensional fast wavelet transform. Step $3 a$ is a change of basis which theoretical complexity is linear, thus the theoretical complexity of the decomposition phase is about $O(N)$. As the same, Step $3 b$ is a change of basis, Steps $2 b$ and Step $3 b$ correspond to an inverse two dimensional fast wavelet transform, the theoretical complexity of recomposition phase is also about $O(N)$.
5.3 Divergence-free Wavelet-based Flow Estimation

The estimation method developed in this section provides a solution to the optical flow estimation problem subject to a divergence-free constraint. The proposed approach falls within the context of wavelet-based multiresolution methods [4] sketched in the previous section. Then, the velocity field $\mathbf{u}$ is searched in terms of its divergence-free wavelet projection (33)

$\mathbf{u}(\mathbf{x})=\sum_{\mathbf{j}, \mathbf{k} \in \mathbb{Z}^{2}} \mathbf{d}_{\mathbf{j}, \mathbf{k}}^{d i v} \boldsymbol{\Psi}_{\mathbf{j}, \mathbf{k}}^{d i v}(\mathbf{x})$,

and its estimation is reduced to the estimation of its divergence-free wavelet coefficients. At a scale $j$, let us adopt the notation:

$$
I_{1}\left(\mathbf{x}, \mathbf{d}_{j}^{d i v}\right)=I_{1}\left(\mathbf{x}+\sum_{|\mathbf{j}| \leq j, \mathbf{k} \in \mathbb{Z}^{2}} \mathbf{d}_{\mathbf{j}, \mathbf{k}}^{d i v} \Psi_{\mathbf{j}, \mathbf{k}}^{d i v}(\mathbf{x})\right) .
$$

The $\mathbf{d}_{j}^{d i v}$ coefficients are hence defined as the minimizers of the objective function:

$F_{d}\left(\mathbf{d}_{j}^{d i v}\right)=\frac{1}{2} \int_{\mathbb{R}^{2}}\left[I_{1}\left(\mathbf{x}, \mathbf{d}_{j}^{d i v}\right)-I_{0}(\mathbf{x})\right]^{2} d \mathbf{x}$,

where $\mathbf{d}_{j}^{d i v}$ is now defined as the set of the divergencefree wavelets coefficients $\left\{\mathbf{d}_{\mathbf{j}, \mathbf{k}}^{\text {div }}\right\}$. Optimization is carried out by a quasi-Newton method (LBFGS), where a BFGS approximation of the Hessian relying solely on the current gradient is handled. The optimal gradient step is in addition given in the sense of the strong Wolf conditions [18]. Obviously, besides the evaluation of the functional $F_{d}$, the descent optimization algorithm requires the computation of its gradient at each iteration step. These computations also are facilitated by the wavelet formulation. As a matter of fact, the gradient $\nabla F_{d}\left(\mathbf{d}_{j}^{d i v}\right)$ corresponds to:

$\nabla F_{d}=\int_{\mathbb{R}^{2}}\left[I_{1}\left(\mathbf{x}, \mathbf{d}_{j}^{d i v}\right)-I_{0}(\mathbf{x})\right] \nabla I_{1}\left(\mathbf{x}, \mathbf{d}_{j}^{d i v}\right) \cdot \Psi_{\mathbf{j}, \mathbf{k}}^{d i v}(\mathbf{x}) d \mathbf{x}$,

Let $\nabla I_{x_{1}}\left(\mathbf{x}, \mathbf{d}_{j}^{d i v}\right)$ and $\nabla I_{x_{2}}\left(\mathbf{x}, \mathbf{d}_{j}^{d i v}\right)$ denote the scalar components of vector $\left[I_{1}\left(\mathbf{x}, \mathbf{d}_{j}^{d i v}\right)-I_{0}(\mathbf{x})\right] \nabla I_{1}\left(\mathbf{x}, \mathbf{d}_{j}^{d i v}\right)$ :

$\nabla I_{x_{1}}\left(\mathbf{x}, \mathbf{d}_{j}^{d i v}\right)=\left[I_{1}\left(\mathbf{x}, \mathbf{d}_{j}^{d i v}\right)-I_{0}(\mathbf{x})\right] \partial_{x_{1}} I_{1}\left(\mathbf{x}, \mathbf{d}_{j}^{d i v}\right)$,

and

$\nabla I_{x_{2}}\left(\mathbf{x}, \mathbf{d}_{j}^{d i v}\right)=\left[I_{1}\left(\mathbf{x}, \mathbf{d}_{j}^{d i v}\right)-I_{0}(\mathbf{x})\right] \partial_{x_{2}} I_{1}\left(\mathbf{x}, \mathbf{d}_{j}^{d i v}\right)$.

Since,

$$
\begin{aligned}
\nabla I_{1}\left(\mathbf{x}, \mathbf{d}_{j}^{d i v}\right) \cdot \Psi_{\mathbf{j}, \mathbf{k}}^{d i v}(\mathbf{x}) & =\partial_{x_{1}} I_{1}\left(\mathbf{x}, \mathbf{d}_{j}^{d i v}\right) \psi_{j_{1}, k_{1}}^{1} \otimes\left(\psi_{j_{2}, k_{2}}^{1}\right)^{\prime} \\
& -\partial_{x_{2}} I_{1}\left(\mathbf{x}, \mathbf{d}_{j}^{d i v}\right)\left(\psi_{j_{1}, k_{1}}^{1}\right)^{\prime} \otimes \psi_{j_{2}, k_{2}}^{1}
\end{aligned}
$$

and

$\left(\psi_{j, k}^{1}\right)^{\prime}=2^{j+2} \psi_{j, k}^{0}$, 
the computation of $\nabla F_{d}\left(\mathbf{d}_{j}^{d i v}\right)$ thus reduces to a simple linear combination of two sets of scalar coefficients obtained by a two dimensional fast wavelet transforms [16]: the scalar wavelet coefficients of $\nabla I_{x_{1}}\left(\mathbf{x}, \mathbf{d}_{j}^{d i v}\right)$, in the wavelet basis generated by $\psi_{j_{1}, k_{1}}^{1} \otimes \psi_{j_{2}, k_{2}}^{0}$, and those of $\nabla I_{x_{2}}\left(\mathbf{x}, \mathbf{d}_{j}^{d i v}\right)$, in the wavelet basis generated by $\psi_{j_{1}, k_{1}}^{0} \otimes \psi_{j_{2}, k_{2}}^{1}$. Precisely, let $\tilde{\mathbf{d}}_{\mathbf{j}, \mathbf{k}}^{1}$ and $\tilde{\mathbf{d}}_{\mathbf{j}, \mathbf{k}}^{2}$ be the set of these coefficients:

$\tilde{\mathbf{d}}_{\mathbf{j}, \mathbf{k}}^{1}=\int_{\mathbb{R}^{2}} \nabla I_{x_{1}}\left(\mathbf{x}, \mathbf{d}_{j}^{d i v}\right) \psi_{j_{1}, k_{1}}^{1} \otimes \psi_{j_{2}, k_{2}}^{0} d \mathbf{x}$,

$\tilde{\mathbf{d}}_{\mathbf{j}, \mathbf{k}}^{2}=\int_{\mathbb{R}^{2}} \nabla I_{x_{2}}\left(\mathbf{x}, \mathbf{d}_{j}^{d i v}\right) \psi_{j_{1}, k_{1}}^{0} \otimes \psi_{j_{2}, k_{2}}^{1} d \mathbf{x}$,

we get:

$\nabla F_{d}\left(\mathbf{d}_{j}^{d i v}\right)=\left[2^{j_{2}+2} \tilde{\mathbf{d}}_{\mathbf{j}, \mathbf{k}}^{1}-2^{j_{1}+2} \tilde{\mathbf{d}}_{\mathbf{j}, \mathbf{k}}^{2}\right]$

Remark 2

The coefficients used in the computation of $\nabla F_{d}\left(\mathbf{d}_{j}^{\text {div }}\right)$ correspond to the dual scalar wavelet bases. To be more precise, according to (15), by definition coefficients $\tilde{\mathbf{d}}_{\mathbf{j}, \mathbf{k}}^{1}$ correspond to the decomposition of $\nabla I_{x_{1}}\left(\mathbf{x}, \mathbf{d}_{j}^{\text {div }}\right)$ on the scalar basis generated by $\left\{\tilde{\psi}_{j_{1}, k_{1}}^{1} \otimes \tilde{\psi}_{j_{2}, k_{2}}^{0}\right\}$ and $\tilde{\mathbf{d}}_{\mathbf{j}, \mathbf{k}}^{2}$ correspond to the decomposition of $\nabla I_{x_{2}}\left(\mathbf{x}, \mathbf{d}_{j}^{\text {div }}\right)$ on the scalar basis generated by $\left\{\tilde{\psi}_{j_{1}, k_{1}}^{0} \otimes \tilde{\psi}_{j_{2}, k_{2}}^{1}\right\}$.

As in the standard wavelet method, to estimate the motion at scale $j<J$, starting with initialization coefficients $\mathbf{d}_{j}^{d i v}$ of length $2^{j} \times 2^{j}$, one step of the optimization procedure requires to:

1c. Compute $\mathbf{u}_{j}$ at fine grid points by extrapolation.

2c. Compute $I_{1}\left(\mathbf{x}+\mathbf{u}_{j}\right)$ using spline interpolation.

3c. Evaluate $F_{d}\left(\mathbf{d}_{j}^{d i v}\right)$ on $\mathbf{u}_{j}$ using quadrature formula.

4c. Compute $\nabla I_{1}\left(\mathbf{x}+\mathbf{u}_{j}\right)$ with finite difference method.

5c. Compute the gradient $\nabla F_{d}\left(\mathbf{d}_{j}^{d i v}\right)$.

Step 1c uses the inverse fast divergence-free wavelet transform, to compute $\mathbf{u}_{j}$ at fine grid points, from its divergence-free wavelets coefficients (see Section 5.2). Step 5c uses (41). Thus, the theoretical complexity of the algorithm is also $O(N)$.

The main advantage of this divergence-free wavelet method, compared to the standard wavelet method, is that it reduces the solution space to a specific subspace respecting the imposed constraint. In addition, the number of degrees of freedom is divided by two: the length of $\left[\mathbf{d}_{j}^{d i v}\right]$ is $2^{j} \times 2^{j}$, instead of $2 \times 2^{j} \times 2^{j}$. Obviously, at fine resolution this method faces the same limitation as any basis with respect to the aperture problem.

\section{High-Order Regularization}

The resolution of inverse problems is complicated by the fact that these problems are generally ill-conditioned. It is therefore necessary to add constraints that reduce the space of possibilities to achieve a unique solution. In this section, we are interested by constraints on the regularity of the solution, expressed through high-order differentiation operators. Without loss of generality, we describe methods for computing these operators effectively using wavelet bases, in the case of optical flow estimation.

Optical flow estimation is one class of inverse problem. Morever, as mentioned earlier equation (2) is a scalar constraint involving two unknowns $u_{1}$ and $u_{2}$. Following [4], in Sections 4 and Section 5, we have shown that truncating at small scales the wavelet expansion of the solution $\mathbf{u}$ yields to interesting coarse scale polynomial approximations of the solution. Nevertheless, in the context of optical flow and especially in the context of fluid motion, the accurate estimation at small scales constitutes a crucial issue. A common approach is to introduce some prior knowledge on the solution regularity $[10,26]$. Subsequently, the objective is to investigate this technique in the context of standard wavelet-based method and divergence-free wavelet-based method.

To make well-conditioned the problem of optimization with the functional defined in (3), one adds a convex regularization term $F_{r}$. The objective function $F_{d}$ given by (3) is then replaced by:

$F(\mathbf{u})=F_{d}(\mathbf{u})+\gamma F_{r}(\mathbf{u})$,

with the parameter $\gamma>0$ that balances data and regularization terms. The value of this weight needs to be adapted, which might be a non-trivial task in practice.

In the following, we focus on three different highorder regularizers terms $F_{r}(\mathbf{u})$. Some of them have proven to be particularly adapted to fluid flows [9]. They are all based on the quadratic penalization of high-order derivatives.

A first possibility is to penalize discrepancies of the velocity field in each direction from a polynomial of 
degree $n$ (i.e penalize derivative of order $n \in \mathbb{N}^{*}$ ):

$F_{r}(\mathbf{u})=\int_{\mathbb{R}^{2}} \sum_{\substack{1 \leq i \leq 2 \\ 1 \leq \ell \leq 2}}\left|\partial_{x_{i}}^{n} u_{\ell}(\mathbf{x})\right|^{2} d \mathbf{x}$.

Other approaches consist in favoring coherent vortex blobs by a second-order curl regularization:

$F_{r}(\mathbf{u})=\int_{\mathbb{R}^{2}}\left|\nabla\left(\partial_{x_{2}} u_{1}(\mathbf{x})-\partial_{x_{1}} u_{2}(\mathbf{x})\right)\right|^{2} d \mathbf{x}$,

or approaching solutions of the heat equation:

$F_{r}(\mathbf{u})=\int_{\mathbb{R}^{2}} \sum_{1 \leq \ell \leq 2}\left|\Delta u_{\ell}(\mathbf{x})\right|^{2} d \mathbf{x}$.

One can notice that regularizers (44) and (45) become identical in the case of divergence-free flows. Indeed, there exist a stream function $\chi$ such as:

$\mathbf{u}=\operatorname{curl} \chi$, with $\chi \in H^{1}\left(\mathbb{R}^{2}\right)$.

From the definition of curl operator in 2D, one can prove that:

$-\Delta \chi=\operatorname{curl}(\mathbf{u})$

and this implies:

$|\nabla \operatorname{curl}(\mathbf{u})|^{2}=|-\nabla \Delta \chi|^{2}=\left|\partial_{x_{1}} \Delta \chi\right|^{2}+\left|\partial_{x_{2}} \Delta \chi\right|^{2}$.

Using again equation (46), we obtain:

$|\Delta(\mathbf{u})|^{2}=|\Delta(\operatorname{curl} \chi)|^{2}=\left|\partial_{x_{1}} \Delta \chi\right|^{2}+\left|\partial_{x_{2}} \Delta \chi\right|^{2}$.

In most classical methods, the operators of differentiation that appear in the previous regularizers (43), (44) and (45) are evaluated using finite difference methods. This leads to complex advanced discrete schemes that must be carefully designed to avoid numerical instabilities or oscillations.

The wavelet context offers an ideal setting to make such computation accurately with less effort. In this paper, we present two different wavelet-based schemes for high-order regularization of inverse problems. Both regularization schemes do not rely on any finite difference discrete approximation of spatial derivatives. The first approach, which is described in section 6.1, is a discrete approximation of regularization integrals. It presents the advantage to be intrinsically very simple since regularization is achieved by penalization of a subset of wavelet coefficients. The second scheme, which is described in section 6.2 , constitutes a very interesting approach since it enables the exact computation of continuous regularization integrals without much more effort. In the two cases, the underlying algorithms have the same theoretical complexity.
6.1 Operator Discrete Approximation Method

In this section we describe an intuitive discrete approximation of the high-order regularization integral (43). The method is based upon differentiation properties of wavelets.

In Proposition 1 we see that the derivative of a regular wavelet basis is another wavelet basis, with analogous properties of $L^{2}(\mathbb{R})$ signal decomposition and reconstruction. Then, for $n \in \mathbb{N}^{*}$ and sufficiently smooth biorthogonal wavelet generators $(\psi, \tilde{\psi})$, let $(\theta, \tilde{\theta})$ be the biorthogonal wavelet generators obtained by applying successively $n$ time the relations of equation (25):

$\frac{d^{n}}{d x^{n}} \psi=4^{n} \theta \quad$ and $\quad \frac{d^{n}}{d x^{n}} \tilde{\theta}=(-4)^{n} \tilde{\psi}$.

For any smooth function $f \in L^{2}(\mathbb{R})$, we have:

$\frac{d^{n}}{d x^{n}} f(x)=\sum_{j, k \in \mathbb{Z}} \bar{d}_{j, k} \theta_{j, k}(x), \quad \bar{d}_{j, k}=<\frac{d^{n}}{d x^{n}} f, \tilde{\theta}_{j, k}>$.

Since $\left\{\theta_{j, k}\right\}_{j, k \in \mathbb{Z}}$ is a Riesz basis of $L^{2}(\mathbb{R})$, we have:

$\left\|\frac{d^{n}}{d x^{n}} f\right\|_{L^{2}(\mathbb{R})} \sim\left\|\bar{d}_{j, k}\right\|_{\ell^{2}\left(\mathbb{Z}^{2}\right)}$

Formally, using integration by part and relation (50), we get:

$\int_{\mathbb{R}} \frac{d^{n}}{d x^{n}} f(x) \tilde{\theta}_{j, k}(x) d x=(-4)^{n} 2^{n j} \int_{\mathbb{R}} f(x) \tilde{\psi}_{j, k}(x) d x$.

Setting $d_{j, k}=<f, \tilde{\psi}_{j, k}>$, one obtains:

$\bar{d}_{j, k}=(-4)^{n} 2^{n j} d_{j, k}$,

thus

$\left\|\frac{d^{n}}{d x^{n}} f\right\|_{L^{2}(\mathbb{R})} \sim\left\|(-4)^{n} 2^{n j} d_{j, k}\right\|_{\ell^{2}\left(\mathbb{Z}^{2}\right)}$.

Since the computation of coefficient $d_{j, k}=<f, \tilde{\psi}_{j, k}>$ involves only $f$ instead of $\frac{d^{n}}{d x^{n}} f$, the penalization of wavelet coefficients' amplitude thus enables to control the amplitude of the derivative of the estimated signal. A proof of previous formal calculations can be found in [13] and the results can be extended easily to the case of $2 \mathrm{D}$ signals.

To control derivatives of motion components in the neighborhood of points in $\Omega \subset \mathbb{R}^{2}$, which is defined as the set of translation at the finest scale of the dyadic discrete wavelet decomposition, one can use (51):

$F_{r}(\mathbf{u}) \sim \sum_{j, \mathbf{k}} 4^{n j}\left|d_{j, \mathbf{k}}^{\ell}\right|^{2}, \quad \ell=1,2$, 
where $d_{j, \mathbf{k}}^{\ell}$ denotes the set of wavelet coefficients of $u_{\ell}$ : $\left\langle u_{\ell}(\mathbf{x}), \tilde{\varphi}_{j, k_{1}} \otimes \tilde{\psi}_{j, k_{2}}(\mathbf{x})\right\rangle,\left\langle u_{\ell}(\mathbf{x}), \tilde{\psi}_{j, k_{1}} \otimes \tilde{\varphi}_{j, k_{2}}(\mathbf{x})\right\rangle$ and $\left\langle u_{\ell}(\mathbf{x}), \psi_{j, k_{1}} \otimes \psi_{j, k_{2}}(\mathbf{x})\right\rangle$.

Since each coefficient is weighted by $4^{n j}$ in (52), the term $F_{r}(\mathbf{u})$ can be thus expressed in quadratic form:

$F_{r}(\mathbf{u}) \sim \mathbf{d}^{T} \Lambda_{j} \mathbf{d}$,

where $\Lambda_{j}$ is a diagonal matrix, whose entries are $4^{n j}$ and $\mathbf{d}$ the vector of wavelet coefficients $\left\{d_{j, \mathbf{k}}^{\ell}\right\}_{\ell=1,2}$. Then, the gradient of $F_{r}(\mathbf{u})$ according to $\mathbf{d}$ corresponds to:

$\nabla F_{r}(\mathbf{d}) \sim \Lambda_{j} \mathbf{d}$.

\subsection{Operator Continuous Approximation Method}

The great advantage brought by the continuous optical flow representation with a finite set of coefficients of sufficiently "regular" wavelets, is that computation is done on the basis functions. More precisely, it enables the exact calculation of continuous spatial derivatives appearing in $F_{r}$, and the exact computation of the integrals and their gradients. This becomes possible since one knows how to compute exactly the elements of mass and stiffness matrices of compactly supported wavelet basis. To this end, we use the following results proved by Beylkin [2]:

\section{Proposition 2}

Let $\varphi \in L^{2}(\mathbb{R})$ be a scaling function. The function $I_{\varphi}$ of its autocorrelation at a point $x$ defined by:

$I_{\varphi}(x)=\int_{\mathbb{R}} \varphi(y) \varphi(y-x) d y$,

satisfies a two scales relation:

$I_{\varphi}(x)=\sum_{k \in \mathbb{Z}} i_{k} I_{\varphi}(2 x-k)$,

where

$i_{k}=\sum_{\ell \in \mathbb{Z}} h_{\ell} h_{\ell-k}$.

Similarly, the function $J_{\varphi}$ of the correlation of $\varphi$ and its derivative of order $n$ at a point $x$, defined by:

$J_{\varphi}(x)=\int_{\mathbb{R}} \varphi(y) \varphi^{(n)}(y-x) d y$,

also satisfies a two scales relation:

$J_{\varphi}(x)=\sum_{k \in \mathbb{Z}} j_{k} J_{\varphi}(2 x-k)$, with $j_{k}=2^{n} i_{k}$.

In addition, values of $J_{\varphi}$ on integer points verify:

$\sum_{\ell \in \mathbb{Z}} \ell^{n} J_{\varphi}(\ell)=(-1)^{n} n !$
By proposition 2, the inner products of the form $<\varphi_{j, k}, \varphi_{j, k^{\prime}}>$ and $<\varphi_{j, k}^{(n)}, \varphi_{j, k^{\prime}}^{(n)}>$ are eigenvectors of the matrices of terms $i_{k}$ and $j_{k}$ respectively. In practice, these terms are computed by solving an eigenvalue problem if the scaling function $\varphi \in L^{2}(\mathbb{R})$ is compactly supported. To get the wavelet inner products $<\psi_{j, k}, \psi_{j, k^{\prime}}>$ or $<\psi_{j, k}^{(n)}, \psi_{j, k^{\prime}}^{(n)}>$, it suffices to use the two scales relation satisfied by the wavelet $\psi$ to return to the scaling function basis.

Once we can compute the mass matrix and stiffness matrix of a wavelet basis, the computation of the regularization term $F_{r}$ becomes easy. In order to clarify these points, let us explicit the computation of the term $\int_{\mathbb{R}^{2}}\left|\partial_{x_{1}}^{n} u_{1}\right|^{2} d x$ in the case of anisotropic divergence-free wavelet-based method, the other terms being treated similarly. From the definition of the divergence-free wavelets, we obtain:

$u_{1}=\sum_{\mathbf{j}, \mathbf{k} \in \mathbb{Z}^{2}} 2^{j_{2}+2} \mathbf{d}_{\mathbf{j}, \mathbf{k}}^{d i v} \psi_{j_{1}, k_{1}}^{1} \otimes \psi_{j_{2}, k_{2}}^{0}$.

Thus:

$\partial_{x_{1}}^{n} u_{1}=\sum_{\mathbf{j}, \mathbf{k} \in \mathbb{Z}^{2}} 2^{j_{2}+2} \mathbf{d}_{\mathbf{j}, \mathbf{k}}^{d i v} \partial_{x_{1}}^{n} \psi_{j_{1}, k_{1}}^{1} \otimes \psi_{j_{2}, k_{2}}^{0}$,

and

$$
\begin{aligned}
\int_{\mathbb{R}^{2}}\left|\partial_{x_{1}}^{n} u_{1}\right|^{2} d x & =\int_{\mathbb{R}^{2}} \partial_{x_{1}}^{n} u_{1} \cdot \partial_{x_{1}}^{n} u_{1} d x \\
& =\sum \mathbf{d}_{\mathbf{j}, \mathbf{k}}^{d i v} \mathbf{d}_{\mathbf{j}^{\prime}, \mathbf{k}^{\prime}}^{d i v} \mathbf{R}_{k_{1}, k_{1}^{\prime}}^{j_{1}, j^{\prime}} \mathbf{M}_{k_{2}, k_{2}^{\prime}}^{j_{2}, j^{\prime}},
\end{aligned}
$$

where $\mathbf{M}$ and $\mathbf{R}$ are respectively the one dimensional mass and stiffness matrices of the basis $\left\{\psi_{j, k}^{0}\right\}$ and $\left\{\psi_{j, k}^{1}\right\}$. Accordingly, their coefficients are given by:

$$
\begin{aligned}
& \mathbf{M}_{k_{2}, k_{2}^{\prime}}^{j_{2}, j_{2}^{\prime}}=2^{j_{2}+j_{2}^{\prime}+4}\left\langle\psi_{j_{2}, k_{2}}^{0}, \psi_{j_{2}^{\prime}, k_{2}^{\prime}}^{0}\right\rangle, \\
& \mathbf{R}_{k_{1}, k_{1}^{\prime}}^{j_{1}, j_{1}^{\prime}}=\left\langle\frac{d^{n}}{d x^{n}} \psi_{j_{1}, k_{1}}^{1}, \frac{d^{n}}{d x^{n}} \psi_{j_{1}^{\prime}, k_{1}^{\prime}}^{1}\right\rangle .
\end{aligned}
$$

As (62) is a quadratic form, its gradient is simply given by:

$\partial_{\mathbf{d}_{\mathbf{j}, \mathbf{k}}^{d i v}} \int_{\mathbb{R}^{2}}\left|\partial_{x_{1}}^{n} u_{1}\right|^{2} d x=\sum_{\mathbf{j}^{\prime}, \mathbf{k}^{\prime} \in \mathbb{Z}^{2}} \mathbf{d}_{\mathbf{j}^{\prime}, \mathbf{k}^{\prime}}^{d i v} \mathbf{R}_{k_{1}, k_{1}^{\prime}}^{j_{1}, j_{1}^{\prime}} \mathbf{M}_{k_{2}, k_{2}^{\prime}}^{j_{2}, j_{2}^{\prime}}$

To compute the regularization term, we use the tensor structure of the basis, unlike [25] where the twodimensional basis functions are used. This allows us avoiding the calculation and storage of a large matrix, hence reducing greatly the complexity of the algorithm:

$\left[\partial_{\mathbf{d}_{\mathbf{j}, \mathbf{k}}^{d i v}} \int_{\mathbb{R}^{2}}\left|\partial_{x_{1}}^{n} u_{1}\right|^{2} d x\right]=\mathbf{R}\left[\mathbf{d}_{\mathbf{j}, \mathbf{k}}^{d i v}\right] \mathbf{M}$. 
In addition, the computation of these matrices is done once for all on the scaling functions basis. To come back to the wavelet basis it suffices to use one-dimensional fast wavelet transform (FWT) on each row and column. Thus the theoretical complexity of this gradient computation is at most $O\left(N^{3}\right)$, which is much lower than the $O\left(N^{6}\right)$ complexity of [25].

\section{Remark 3}

Since $\frac{d^{n}}{d x^{n}} \psi_{j, k}(x)=4^{n} 2^{j n} \psi_{j, k}^{(n)}(x)$, the entries of the matrix $\mathbf{R}$ defined by (64) are proportional to $16^{n} 4^{n j}$. Thus, the parameter $\gamma>0$ which matches the effect of the regularization term $F_{r}$ must be small enough to attenuate this weighting.

\section{Numerical Results}

\subsection{Synthetic Images of Turbulence}

In this section, the quality of optical flow estimation is evaluated on two different synthetic image sequences: a sequence of Particle Image Velocimetry (PIV) (referred subsequently as "particle images") and images of an advected and diffused passive scalar (referred subsequently as "scalar images"). Both sequences depict the same bi-dimensional incompressible turbulent flow. The dynamic of the fluid flow is given by a direct numerical simulation of 2D incompressible Navier-Stokes equations at $R e=3000$, using the vorticity conservation equation and the Lagrangian equation for non-heavy particles transported by the flow (simulation details can be found in [8]). Since this simulated flow is divergencefree by construction, we can evaluate the efficiency of introducing this constraint in the wavelet bases. Image size is $256 \times 256$ pixels and the pixel grey levels have been normalized; examples of input images $I_{0}(x)$ from PIV and scalar sequences are displayed in Figure 9, together with their associated ground truth motion vorticity. Estimated velocity and vorticity fields are evaluated based on the Root Mean Squared end-point Error (RMSE) and the Mean Barron Angular Error (MBAE). For the two image sequences, the different realized experiments correspond to:

- Case (i), estimation on a truncated standard wavelet basis [4], without any regularization.

- Case (ii), estimation on a truncated divergence-free wavelet basis, without any regularization.

- Case (iii), estimation on a divergence-free wavelet basis using discrete approximation of the regularization term.

- Case (iv), estimation on a divergence-free wavelet basis using continuous approximation of the regularization term.
On Figure 6, we show the plot of a time-sequence of the RMSE on the velocity field $\mathbf{u}$ obtained by some stateof-the-art estimators and compare it to the velocity obtained with the proposed methods. Only the method of [26] encodes the same regularizer operators (divergencefree and gradient of curl penalization), which are approached by high-order schemes using finite-difference method. The other results are used to compare the capacity and effectiveness of the proposed methods over existing state-of-the-art estimators. Let us note that state-of-the-art PIV techniques based on correlation use largely above these results in term of RMSE (mean value around 0.1 ). For indication, also an advanced implementation of the Horn and Schunck [10] techniques is of the same order accuracy as state-of-the-art PIV method. Figure 7 shows the plot of a time-sequence of RMSE on the vorticity $\omega=\operatorname{curl}(\mathbf{u})$ obtained by the proposed methods, compared to the results of $[7,10$, $26]$.

The divergence-free wavelet generator $\psi^{1}$ (cases (ii), (iii), (iv)) was the Coiflet [16] with 10 vanishing moments. This same Coiflet-10 was used for the standard estimation on truncated basis, case (i), for comparison. For the operator discrete approximation method, case (iii), we used the second order derivative penalization corresponding to:

$$
F_{r}(\mathbf{u})=\int_{\Omega} \sum_{\substack{1 \leq i \leq 2 \\ 1 \leq \ell \leq 2}}\left|\partial_{x_{i}}^{2} u_{\ell}(\mathbf{x})\right|^{2} d \mathbf{x} .
$$

The regularization parameters are $\gamma=2.3 \cdot 10^{-8}$ for the particle images and $\gamma=2 \cdot 1 \cdot 10^{-10}$ for the scalar images. For the operator continuous approximation method, case (iv), we used Laplacian regularization (45), approximated as in Section 6.2. The regularization parameters are $\gamma=2.10^{-7}$ for the particle images and $\gamma=10^{-6}$ for the scalar images.

Figure 6, Figure 7 and Figure 8 show that the use of a divergence-free wavelet basis yields a significant improvement, compared to standard wavelet basis or other state-of-the-art estimators. The addition of, either the discrete or the continuous proposed regularization, enables to further outperform state-of-the-art results. Results on scalar imagery (Figure 6(b)) show that the combination of a divergence-free wavelet basis and continuous operator regularization is necessary, in order to obtain results comparable to those of the stateof-the-art. Let us note that the regularization approach proposed in [7] is accurate here since it takes advantage of an additional physical constraint (turbulence power laws parameters, whose estimation increases sig- 
nificantly the computational cost of the method). This type of regularization is perfectly suited to homogeneous isotropic turbulent flows as the one of this test sequence. It is however not adapted to flow showing different regimes in the same domain (laminar, transition toward turbulence, turbulent). The other estimators are from that point of view more general.

Figure 10 and Figure 11 present vorticity fields computed from estimated motion fields, as well as vorticity end-point error maps. On particle imagery, improvements brought by divergence-free bases (cases (i), (ii)) are visible on error maps (Figure 10(b) and Figure 10(d)), only smallest structures remain unestimated. The benefit of a continuous implementation of the Laplacian regularization instead of discrete derivative penalization (cases (iii), (iv)) can be clearly noted looking a vorticity maps (Figure 10(e) and Figure 10(g)). This is at the more true in the scalar case where the discrete approximation yields clearly some block artifacts. This is confirmed by results on scalar imagery Figure 11.

\subsection{Experimental Turbulent Image Sequences}

This section presents results obtained with real images. In order to study quasi-2D or $3 \mathrm{D}$ turbulent flows, the use of $2 \mathrm{D}$ experimental images is very common in fluid mechanics laboratories. However, traditional motion estimators usually fail or exhibit strong inaccuracy at some places.

The first data set consists in images of dispersion of passive tracers in a forced $2 \mathrm{D}$ turbulence experiment, of size $512 \times 512$ pixels [11]. The experiments were performed with electromagnetically-forced incompressible flows in stable thin stratified layers of fluid. Figure 12 depicts an image of the sequence. For the divergencefree wavelet based-method, the employed regularizer is the same as in the case of synthetic images: continuous gradient of curl regularizer (48) (or equivalently, in this incompressible case, the Laplacian penalization) with a factor $\gamma=2 \cdot 5 \cdot 10^{-8}$. In order to make results comparable, we used the same regularizer model and factor for the standard wavelet-based method, followed by a projection onto the divergence-free function space using a spectral method. Inspection of estimates shows that the divergence-free wavelet-based method enables to extract more accurately vortex structures and shear layers with better temporal continuity. This is illustrated by the plot of two consecutive vorticity maps in Figure 13.

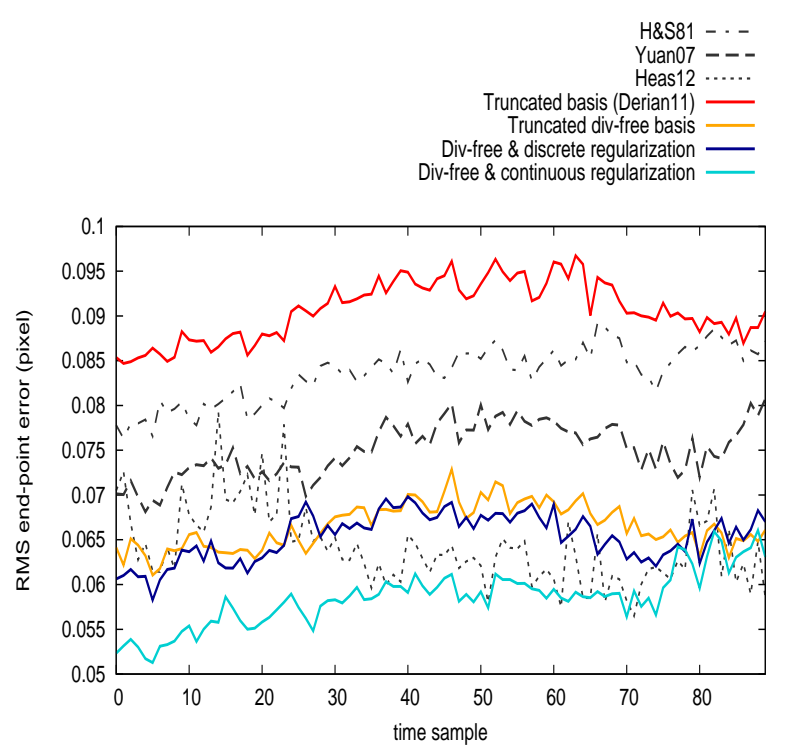

(a) Particles images

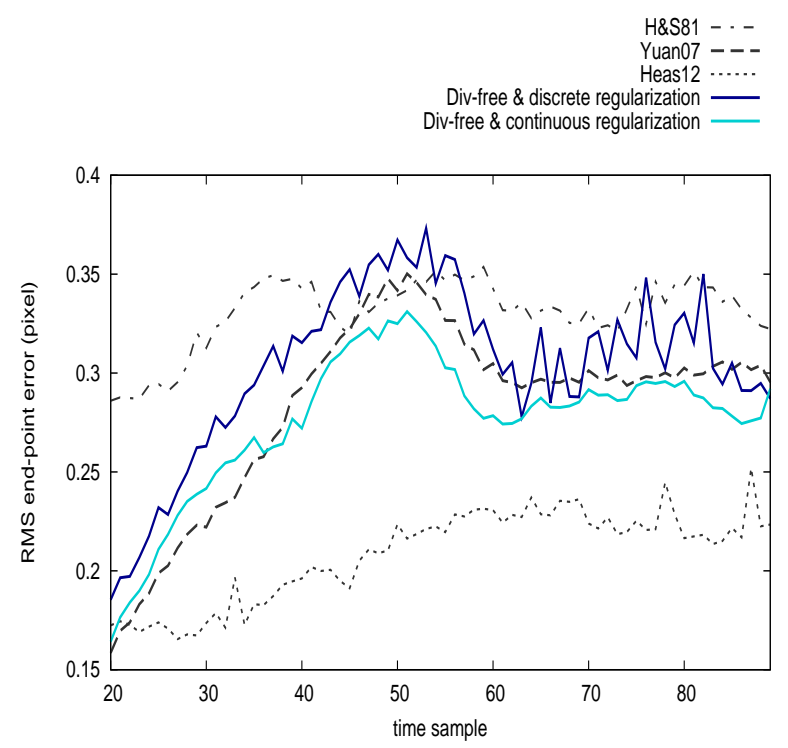

(b) Scalar images

Fig. 6: Comparison of the RMSE on $\mathbf{u}$ between the proposed methods (bold lines) and some works of the literature $[7,10,26]$. Top: from particle images, using truncated basis with usual (case (i), red) or divergence-free (case (ii), orange) wavelets, divergencefree basis with discrete (case (iii), dark blue) or continuous (case (iv), greenish-blue) regularization operators. Bottom: from scalar imagery, using divergence-free basis with discrete (case (iii), dark blue) or continuous (case (iv), greenish-blue) regularization operators.

The second real data set consists in a sequence of 128 images of particle transported by a planar concomitant jet flow, of size $1024 \times 1024$ pixels. The flow is $3 \mathrm{D}$ and shows two high-shear regions featuring development of Kelvin-Helmholtz instabilities. Since the flow 


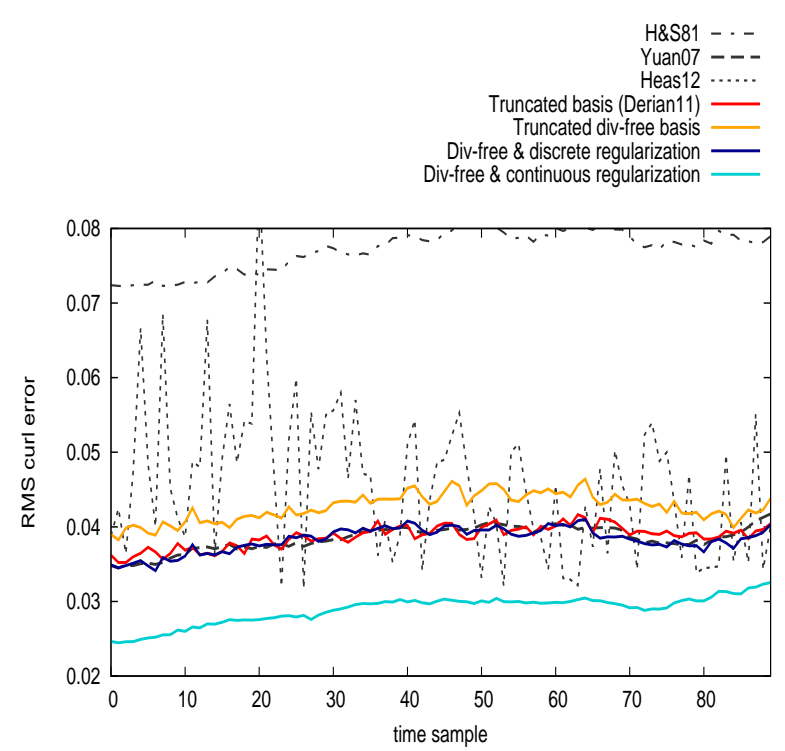

(a) Particles images

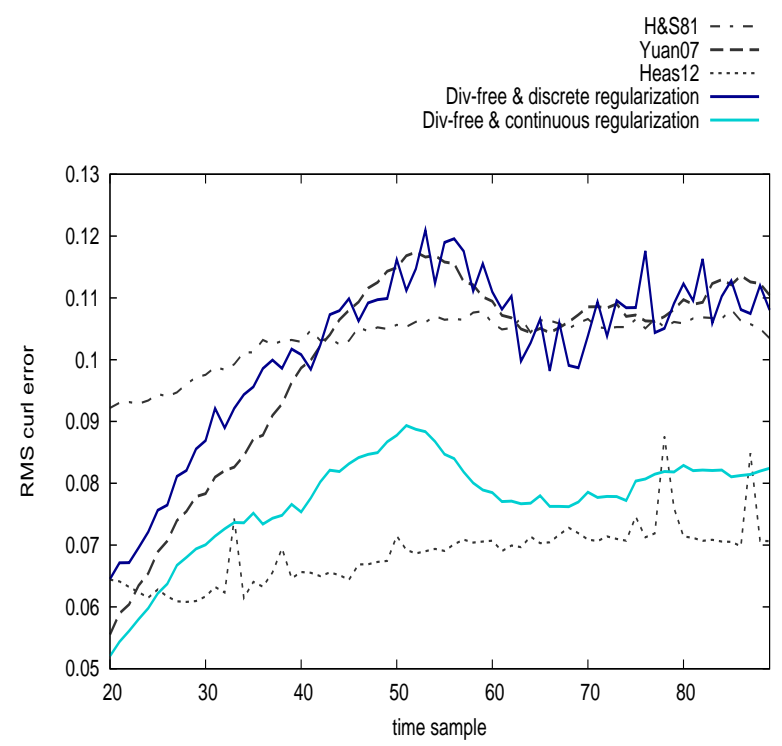

(b) Scalar images

Fig. 7: Comparison of the RMSE on $\omega=\operatorname{curl}(\mathbf{u})$ between the proposed methods (bold lines) and some works of the literature $[7,10,26]$. Top: from particle images, using truncated basis with usual (case (i), red) or divergence-free (case (ii), orange) wavelets, divergence-free basis with discrete (case (iii), dark blue) or continuous (case (iv), greenish-blue) regularization operators. Bottom: from scalar imagery, using divergence-free basis with discrete (case (iii), dark blue) or continuous (case (iv), greenish-blue) regularization operators.

is not divergent-free, motion components are estimated on a standard scalar wavelet basis with the proposed continuous implementation of wavelet-based gradient of curl regularizer (44), using factor $\gamma=10^{-7}$. Results are shown in Figure 14. Estimates obtained with discrete second order regularization (65), with param-

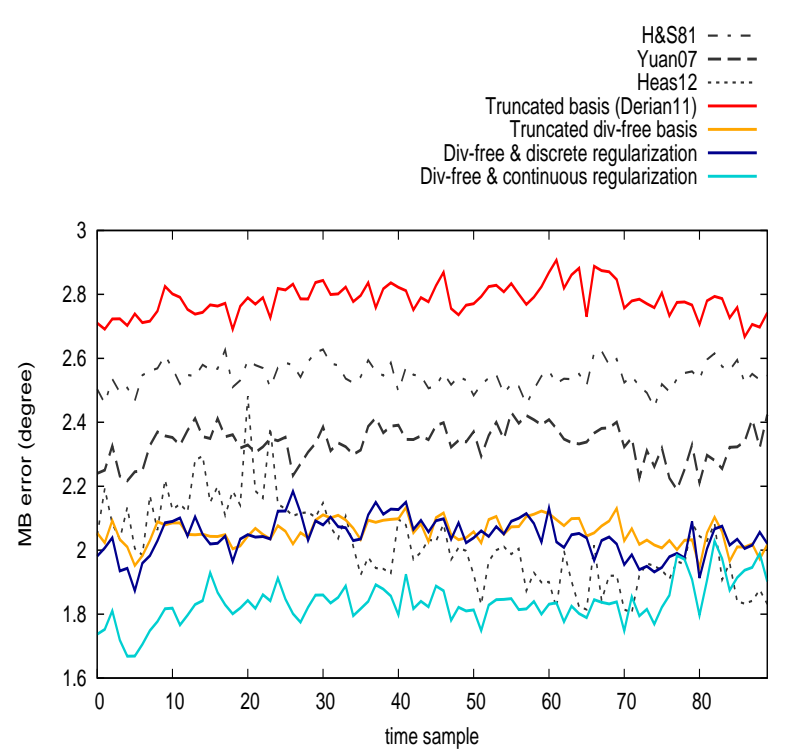

(a) Particles images

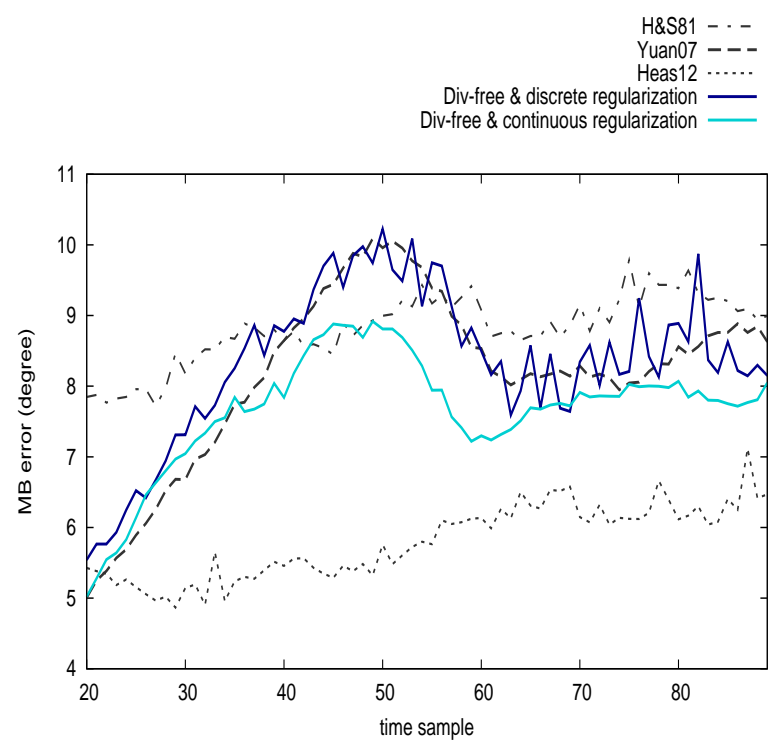

(b) Scalar images

Fig. 8: Comparison of the MBE on $\mathbf{u}$ between the proposed methods (bold lines) and some works of the literature $[7,10,26]$. Top: from particle images, using truncated basis with usual (case (i), red) or divergence-free (case (ii), orange) wavelets, divergencefree basis with discrete (case (iii), dark blue) or continuous (case (iv), greenish-blue) regularization operators. Bottom: from scalar imagery, using divergence-free basis with discrete (case (iii), dark blue) or continuous (case (iv), greenish-blue) regularization operators.

eter $\gamma=10^{7}$, are given for comparison. Figure 14 also presents image of the sequence and streamlines of the estimated velocity field using either discrete or continuous regularization, along with two consecutive vorticity maps computed from estimated motions. A qualitative evaluation of the results shows a remarkably good 

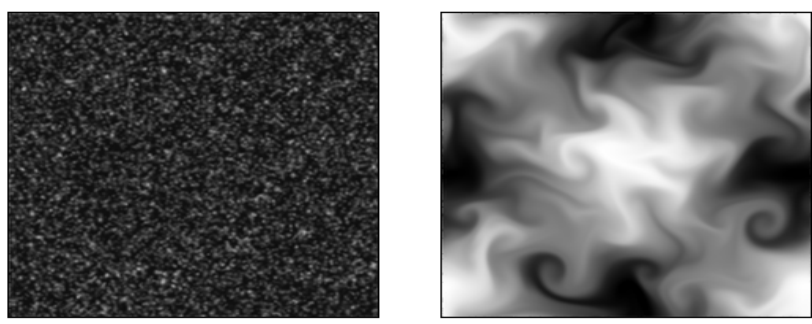

(a) First particle image $I_{0}(x)$

(b) First scalar image $I_{0}(x)$
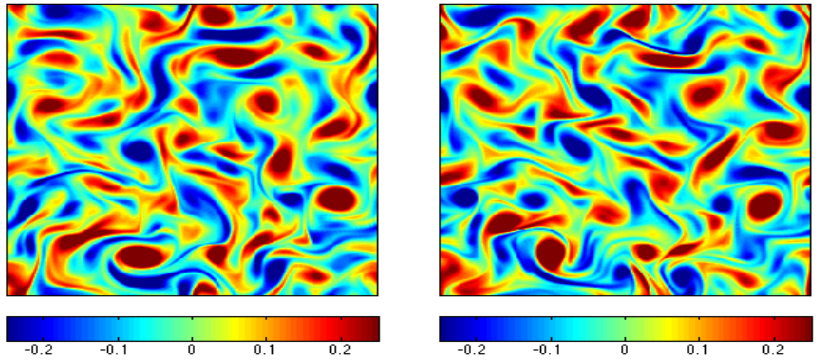

(c) True vorticity, $t=0$ (particle)(d) True vorticity, $t=20$ (scalar)

Fig. 9: Top: first particle image used 9(a) (corresponding to instant $t=0$ in sequence of Fig 6(a)) and first scalar image used 9(b) (corresponding to instant $t=20$ in sequence of Fig. 6(b)). Bottom: first reference vorticity 9(c) (associated to particle images) and first reference vorticity 9(d) (associated to scalar images).

agreement with the physics of concomitant jets. A very good temporal coherence is also observed, although no prior dynamic model is considered (i.e successive pairs of images are processed independently). Results obtained using operator continuous approximation regularization however enables visualizing the evolution of finer structures living at much finer scales.

\section{Conclusion}

Based on a biorthogonal wavelet expansion of optical flow and particularly divergence-free wavelet in the incompressible case, we have proposed an algorithm dedicated to the estimation of fluid motion. The waveletbased algorithm is of low-complexity and offers an intrinsic and efficient multiresolution estimation framework. Taking advantage of the continuous representation of optical flow by a finite set of wavelet coefficients, we have proposed a family of high-order regularizers designed for fluid flows. They rely on the approximation or the exact computation (without any discretization approximations in both cases) of differential operators of

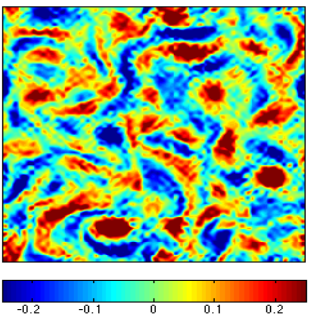

(a) Case (i) vorticity

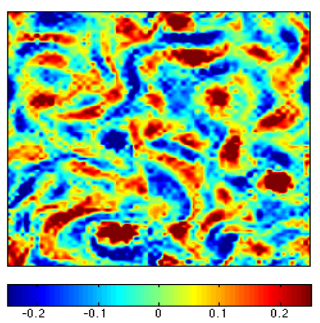

(c) Case (ii) vorticity

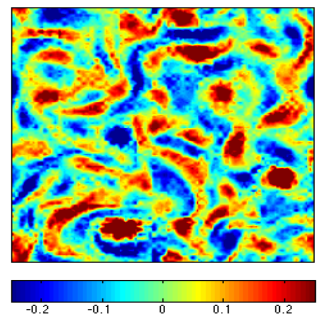

(e) Case (iii) vorticity

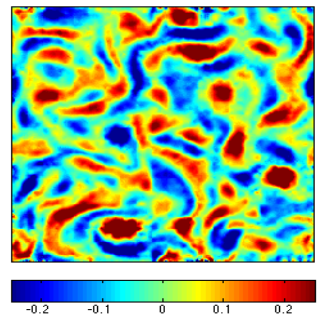

(g) Case (iv) vorticity

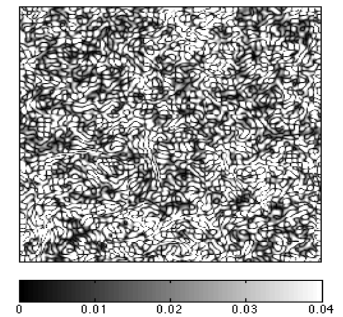

(b) $\mathrm{RMSE}=0.0362$

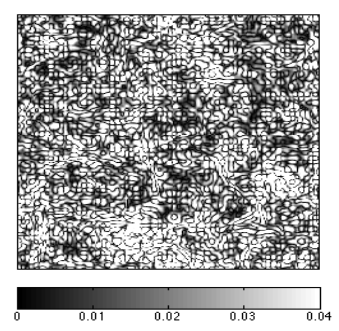

(d) $\mathrm{RMSE}=0.0389$

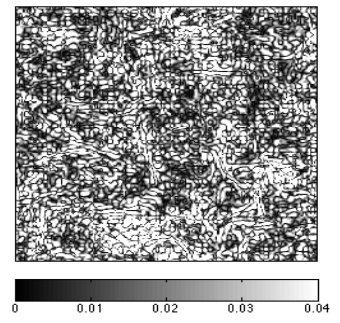

(f) $\mathrm{RMSE}=0.0349$

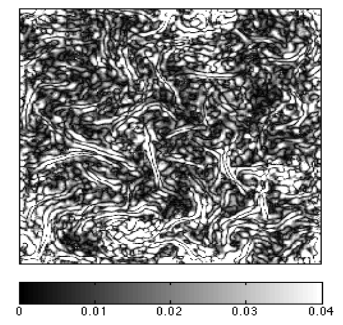

(h) $\mathrm{RMSE}=0.0246$
Fig. 10: Left column: vorticity computed from velocity fields estimated from particle imagery (Fig. 9(a)) with the 4 presented cases, to be compared with the reference Fig. 9(c). Right column: corresponding vorticity RMSE.

arbitrary order. The regularizers are approached in the first case by simply constraining wavelet coefficients, while in the second case it is calculated exactly by the simple calculation of one-dimensional wavelet basis mass and stiffness matrices. Numerical results obtained with challenging particle and scalar image sequences of $2 \mathrm{D}$ and $3 \mathrm{D}$ turbulence show a significant performance enhancement compared to state of the art methods. 


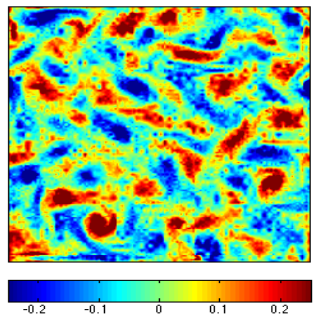

(a) Case (iii) vorticity

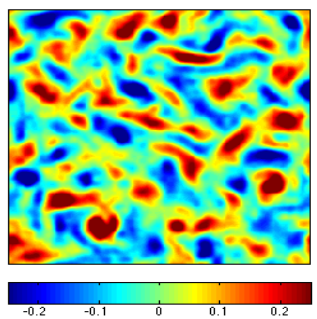

(c) Case (iv) vorticity (b) $\mathrm{RMSE}=0.0644$

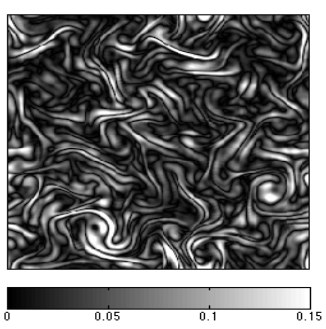

(d) $\mathrm{RMSE}=0.0520$

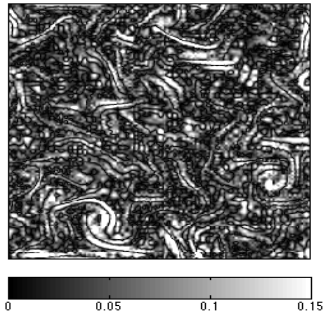

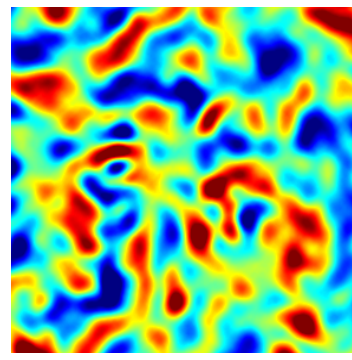

(a) Usual basis, $t=88$.

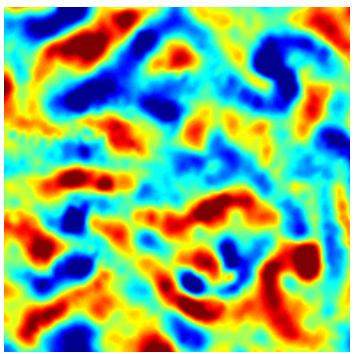

(c) Div.-free basis, $t=88$. (d) Div.-free basis, $t=89$

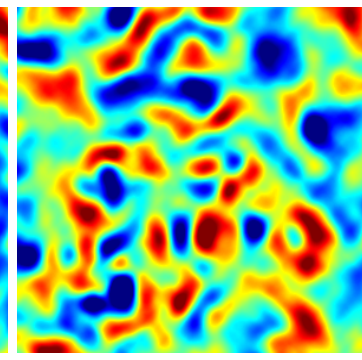

(b) Usual basis, $t=89$.
Fig. 11: Left: vorticity computed from velocity fields estimated from scalar imagery (Fig. 9(b)), using Div-free wavelets method and discrete (top) or continuous (bottom) regularization. Right: corresponding vorticity RMSE.

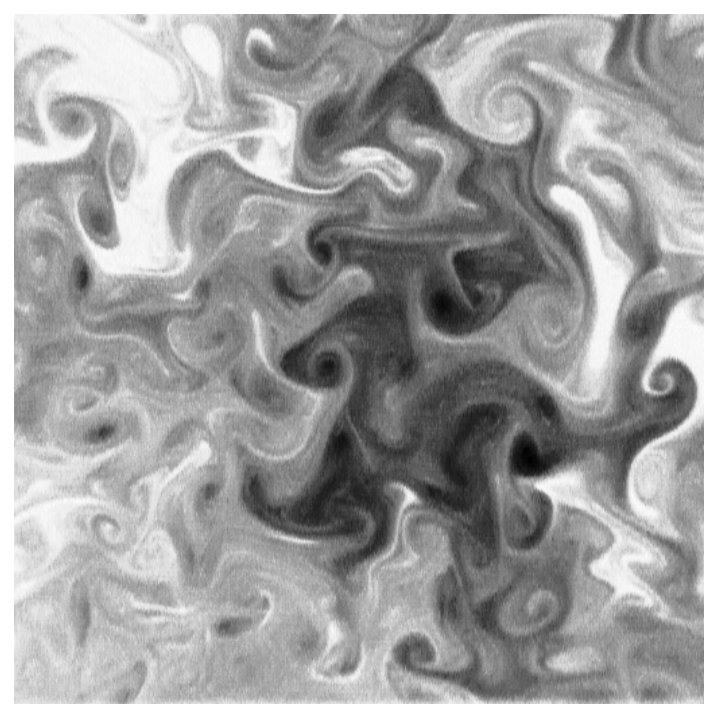

Fig. 12: Experimental image of passive scalar dispersion in a $2 \mathrm{D}$ turbulent motion, corresponding to time $t=89$. This image has been normalized so as to enhance visualization.

\section{References}

1. Bergen, J., Burt, P., Hingorani, R., Peleg, S.: A 3-frame algorithm for estimating two-component image motion. IEEE Trans. Pattern Anal. Mach. Intell. 14(9), 886-895 (1992)

2. Beylkin, G.: On the representation of operator in bases of compactly supported wavelets. SIAM J. Numer. Anal 6(6), $1716-1740$ (1992)
Fig. 13: Details of two successive vorticity fields computed from estimated velocity fields, using experimental 2D scalar image dataset at $t=88$ and $t=89$ (input image Fig. 12). Both cases use continuous Laplacian regularization with $\gamma=2.5 .10^{-8}$, associated to usual Coiflet-10 wavelet basis (top) and divergencefree basis generated from same Coiflet-10 (bottom). Enhancement brought by the divergence-free basis is clearly visible, with much better-defined structures as well as a better temporal coherence on bottom row vorticity fields.

3. Cohen, A., Daubechies, I., Feauveau, J.C.: Bi-orthogonal bases of compactly supported wavelets. Comm. Pure Appli. Maths 45, 485-560 (1992)

4. Dérian, P., Héas, P., Herzet, C., Mémin, E.: Wavelet-based fluid motion estimation. In: 3rd International Conference on Scale-Space and Variational Methods in Computer Vision (SSVM2011), IEEE LNCS. Ein-Gedi, Israel (2011)

5. Deriaz, E., Perrier, V.: Divergence-free and Curl-free wavelets in $2 \mathrm{D}$ and $3 \mathrm{D}$, application to turbulence. J. of Turbulence 7 , $1-37$ (2006)

6. Girault, V., Raviart, P.: Finite Element Methods for NavierStokes Equations: Theory and Algorithms. Springer Series in Computational Mathematics. Springer-Verlag. (1986)

7. Heas, P., Memin, E., Heitz, D., Mininni, P.:Power laws and inverse motion modeling: application to turbulence measurements from satellite images. Tellus A (2012), 64, 10962, DOI: 10.3402/tellusa.v64i0.10962

8. Heitz, D., Carlier, J., Arroyo, G.: Final report on the evaluation of the tasks of the workpackage 2, FLUID project deliverable 5.4. Tech. rep., INRIA - Cemagref (2007)

9. Heitz, D., Mémin, E., Schnörr, C.: Variational fluid flow measurements from image sequences: synopsis and perspectives. Experiments in fluids 48(3), 369-393 (2010)

10. Horn, B., Schunck, B.: Determining optical flow. Artificial Intelligence 17, 185-203 (1981)

11. Jullien, M., Castiglione, P., Tabeling, P.: Experimental Observation of Batchelor Dispersion of Passive Tracers. Physical Review Letters 85(17), 3636-3639 (2000)

12. Kohlberger, T., Mémin, E., Schnörr, C.: Dense Motion Estimation Using the Helmholtz Decomposition. Scale Space Methods in Computer Vision, 2695, 432-448 (2003) 

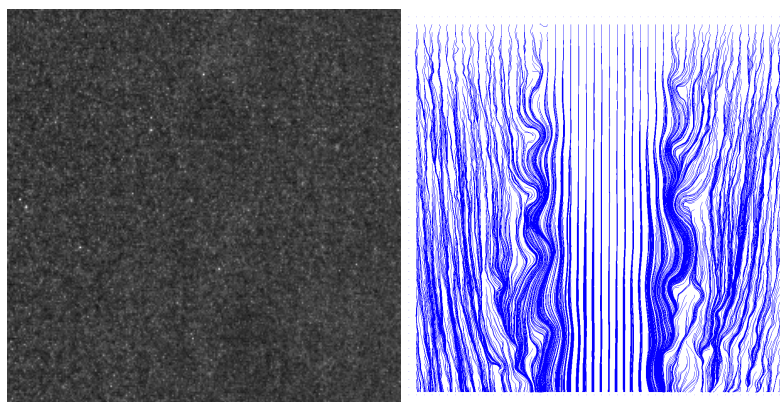

(a) First scalar image $I_{0}(x)$

(b) Example of streamlines

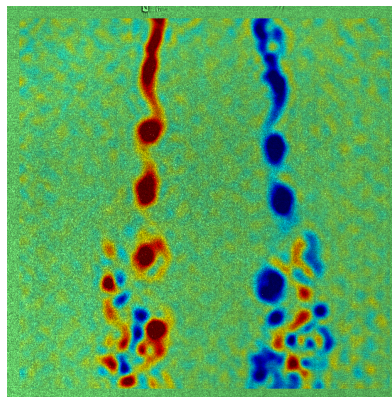

(c) Discrete regul., $\mathrm{t}=16$

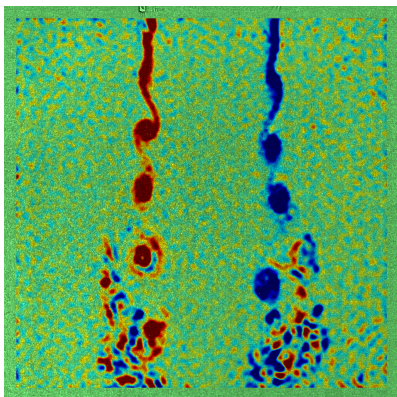

(e) Continuous regul., $\mathrm{t}=16$

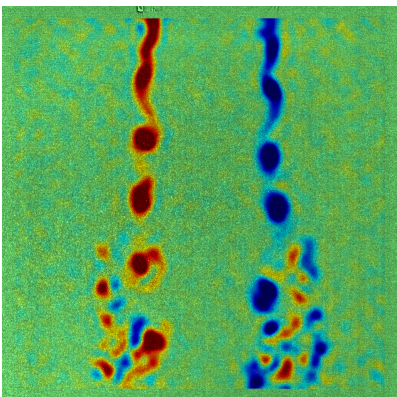

(d) Discrete regul., $\mathrm{t}=17$

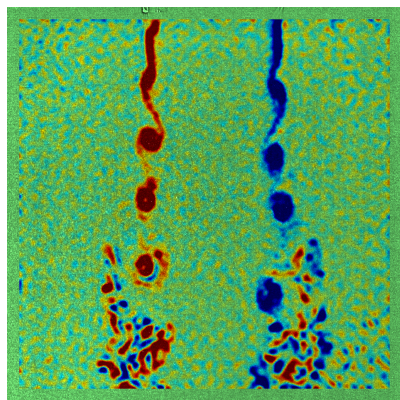

(f) Continuous regul., $\mathrm{t}=17$

Fig. 14: Results from 2D planar jet PIV dataset. Top: detail of input PIV image 14(a), streamlines 14(b). Middle: vorticity computed from two consecutive estimated motions, using discrete regularization. Bottom: vorticity computed from estimations using continuous regularization.

13. Kahane, J.-P., Lemarié-Rieusset, P.-G.: Fourier series and wavelets. Gordon and Breach (1995)

14. Lemarié-Rieusset, P.-G: Analyses multirésolutions non orthogonales, commutation entre projecteurs et dérivation et ondelettes vecteurs à divergence nulle. Revista Matematica Iberoamericana 8, 221-237 (1992)

15. Liu, T., Shen, L.: Fluid flow and optical flow. Journal of Fluid Mechanics 614, 253 -291(2008)

16. Mallat, S.: A Wavelet Tour of Signal Processing: The Sparse Way. Academic Press (2008)

17. Sass-Hansen, M., Larsen, R., Christensen, N.-V.: Curlgradient Image Warping-Introducing Deformation Potentials for Medical Image Registration using Helmholtz Decomposition. VISSAPP 1, 79-185 (2009)

18. Nocedal, J., Wright, S.J.: Numerical Optimization. Springer Series in Operations Research. Springer-Verlag, New York, NY (1999)

19. Papadakis, N., Corpetti, T., Mémin., E: Dynamically consistent optical flow estimation. IEEE International Conference on Computer Vision, Rio de Janeiro, Brasil, (2007)

20. Ruhnau, P., Schnörr, C.: Optical Stokes flow estimation: an imaging-based control approach. Experiments in Fluids $\mathbf{4 2}$ 61-78 (2007)

21. Simard, P.Y., Mailloux, G.E.: A Projection Operator for the Restoration of Divergence-Free Vector Fields. IEEE Transactions on Pattern Analysis and Machine Intelligence 10, 248-256 (1998)

22. Steinbruecker, F., Pock, T., Cremers, D.: Large displacement optical flow computation without warping. In: IEEE International Conference on Computer Vision (ICCV). Kyoto, Japan (2009)

23. Suter, D.: Motion estimation and vector splines. In: Proc. Conf. Comp. Vision Pattern Rec., 939-942. Seattle, USA (1994)

24. Terzopoulos, D.: Regularization of inverse visual problems involving discontinuities. IEEE Transactions on Pattern Analysis and Machine Intelligence 8, 413-424 (1986)

25. Wu, Y., Kanade, T., Li, C., Cohn, J.: Image registration using wavelet-based motion model. Int. J. Computer Vision 38(2), 129-152 (2000)

26. Yuan, J., Schnörr, C., Mémin, E.: Discrete orthogonal decomposition and variational fluid flow estimation. Journ. of Math. Imaging \& Vison 28, 67-80 (2007) 